\title{
SARS-CoV-2 hijacks neutralizing dimeric IgA for enhanced nasal infection and injury
}

Biao Zhou

the University of Hong Kong

Runhong Zhou

University of Hong Kong

Jasper Fuk-Woo Chan

The University of Hong Kong https://orcid.org/0000-0001-6336-6657

Jianwei Zeng

Tsinghua University https://orcid.org/0000-0002-6880-3366

Qi Zhang

Tsinghua University

Shuofeng Yuan

The University of Hong Kong

Li Liu

University of Hong Kong https://orcid.org/0000-0003-2566-7357

Rémy Robinot

Institut Pasteur https://orcid.org/0000-0002-3651-0171

Sisi Shan

Tsinghua University https://orcid.org/0000-0002-7184-6818

Jiwan Ge

Tsinghua University

Hugo Yat-Hei Kwong

The University of Hong Kong

Dongyan Zhou

The University of Hong Kong

Haoran Xu

The University of Hong Kong

\section{Chris Chan}

The University of Hong Kong https://orcid.org/0000-0001-7089-4829

\section{Vincent Poon}

The University of Hong Kong

\section{Hin Chu}

The University of Hong Kong https://orcid.org/0000-0003-2855-9837 
The University of Hong Kong

\section{Ka-Yi Kwan}

The University of Hong Kong

\section{Chun Yin Chan}

AIDS Institute and Department of Microbiology, State Key Laboratory of Emerging Infectious Diseases,

Li Ka Shing Faculty of Medicine, The University of Hong Kong

\section{$\mathrm{Na}$ Liu}

The University of Hong Kong

\section{Chris Chun-Yiu Chan}

The University of Hong Kong

\section{Kenn Ka-Heng Chik}

University of Hong Kong

\section{Zhenglong Du}

The University of Hong Kong

\section{Ka-Kit Au}

The University of Hong Kong

\section{Haode Huang}

University of Hong Kong

\section{Hiu-On Man}

The University of Hong Kong

\section{Jianli Cao}

The University of Hong Kong

\section{Cun Li}

University of Hong Kong

\section{Ziyi Wang}

Tsinghua University

\section{Jie Zhou}

University of Hong Kong https://orcid.org/0000-0002-2948-3873

\section{Youqiang Song}

The University of Hong Kong

\section{Man Lung Yeung}

The University of Hong Kong

\section{Kelvin To}

The University of Hong Kong https://orcid.org/0000-0002-1921-5824

\section{David Ho}

Columbia University Irving Medical Center https://orcid.org/0000-0003-1627-149X

\section{Lisa A. Chakrabarti}

Institute Pasteur

\section{Xinquan Wang}


Tsinghua University https://orcid.org/0000-0003-3136-8070

\section{Linqi Zhang}

Tsinghua University https://orcid.org/0000-0003-4931-509X

\section{Kwok-Yung Yuen}

The University of Hong Kong https://orcid.org/0000-0002-2083-1552

\section{Zhiwei Chen ( $\sim$ zchenai@hku.hk)}

The University of Hong Kong https://orcid.org/0000-0002-4511-2888

\section{Article}

Keywords: SARS-CoV-2, COVID-19, coronavirus, neutralizing antibody, IgG, cryo-EM, mlgA, dlgA, nasal turbinate, antibody-dependent enhancement

Posted Date: October 5th, 2021

DOl: https://doi.org/10.21203/rs.3.rs-923755/v1

License: (c) (i) This work is licensed under a Creative Commons Attribution 4.0 International License. Read Full License 


\section{Abstract}

Robust severe acute respiratory syndrome coronavirus-2 (SARS-CoV-2) infection in nasal turbinate (NT) accounts for high viral transmissibility, yet whether neutralizing IgA antibodies can control it remains unknown. Here, we evaluated receptor binding domain (RBD)-specific monomeric B8-mlgA1 and B8mlgA2, and dimeric B8-dlgA1 and B8-dlgA2 against intranasal SARS-CoV-2 challenge in Syrian hamsters. These antibodies exhibited comparably potent neutralization against authentic virus by competing with human angiotensin converting enzyme-2 (ACE2) receptor for RBD binding. While reducing viruses in lungs, pre-exposure intranasal B8-dlgA1 or B8-dlgA2 led to 81-fold more infectious viruses and severer damage in NT than placebo. Virus-bound B8-dlgA1 and B8-dlgA2 could engage CD209 as an alternative receptor for entry into ACE2-negative cells and allowed viral cell-to-cell transmission. Cryo-EM revealed B8 as a class II neutralizing antibody binding trimeric RBDs in 3-up or 2-up/1-down conformation. Therefore, RBD-specific neutralizing dlgA engages an unexpected action for enhanced SARS-CoV-2 nasal infection and injury in Syrian hamsters.

\section{Introduction}

Severe acute respiratory syndrome coronavirus 2 (SARS-CoV-2), a member of the Betacoronavirus genus, is the causative agent of Coronavirus Disease 2019 (COVID-19) ${ }^{1}$. SARS-CoV-2 enters host cells through the binding of the receptor binding domain (RBD) of its surface trimeric spike (S) protein to the cellular angiotensin-converting enzyme-2 (ACE-2) receptor ${ }^{2-4}$. After the ACE2 binding, the $S$ protein is cleaved into $\mathrm{S} 1$ and $\mathrm{S} 2$ subunits by host cellular proteases including the transmembrane protease serine 2 (TMPRSS2) to promote fusion of viral and cellular membranes for viral entry ${ }^{5-8}$. Apparently, these entry processes are similar to those of SARS-CoV-1 ${ }^{9-12}$, the causative agent of SARS, although these two coronaviruses share just $76 \%$ and $40 \%$ amino acid identity between their genomes and their RBD external subdomains, respectively ${ }^{13,14}$. SARS-CoV-2, however, has displayed remarkably higher transmissibility than SARS-CoV-1. By August 2021, the rapid all-year-round transmission of SARS-CoV-2 has resulted in over 200 million infections and 4 million deaths globally ${ }^{15}$ since the outbreak of COVID-19 reported in December 2019. This is in marked contrast to the SARS epidemic, which caused only 8096 cases and 774 deaths, and disappeared in $2003^{16}$. Therefore, understanding the mechanisms underlying the high transmissibility of SARS-CoV-2 is essential for pandemic control.

The high transmissibility of SARS-CoV-2 is likely associated with multiple factors. First, unlike SARS-CoV1-infected cases characterized by high fever and prominent respiratory symptoms, afebrile individuals with SARS-CoV-2 were often found upon diagnosis, allowing person-to-person transmissions by asymptomatic carriers including international travellers ${ }^{17-20}$. Second, while both coronaviruses employ the same receptor ACE2, highly conserved RBD residues or side chain properties of SARS-CoV-2 might account for increased ACE2 binding ${ }^{9-12}$. Third, the unique insertion of the PRRA sequence in SARS-CoV-2 
S glycoprotein promotes higher virion infectivity and cell-cell fusion, leading to enhanced pathogenicity in vivo $^{21-23}$. Fourth, by altering the S protein conformation, the D614G mutation increases the stability of $S$ trimer to avoid premature S1 shedding, which results in a rapid dominance of this mutation globally 24,25 . The D614G mutation also induces higher infectious titres in nasal washes and the trachea of infected hamsters ${ }^{26}$. Multiple mechanisms, therefore, contribute to the high upper respiratory tract (URT) viral loads characteristic of SARS-CoV-2 infection ${ }^{27-29}$, which facilitates viral transmission in the human population. Till now, the role of nasal IgA remains understudied. In particular, whether antibody-dependent enhancement (ADE) of SARS-CoV-2 infection has any place in vivo remains an open question ${ }^{30}$.

Since the outbreak of COVID-19, worldwide research efforts have led to the identification of many potent human neutralizing antibodies (HuNAbs) mainly in IgG form for preclinical and clinical developments ${ }^{31-}$ 38 . Some studies also investigated IgA antibodies, which are known to play an important role in mucosal immunity, especially in their secretory form (SIgA) ${ }^{39,40}$. RBD-specific IgA antibodies were rapidly discovered in COVID-19 patients ${ }^{41}$. IgA and SIgA were even shown to dominate the early antibody response as compared to IgG and IgM in saliva and bronchoalveolar lavage fluids due to expansion of IgA plasmablasts with mucosal homing characteristics ${ }^{42,43}$. However, it was noted that COVID-19 patients with acute respiratory distress syndrome (ARDS) had higher SIgA in the airway mucosa for unknown reasons ${ }^{44}$. Moreover, a recent study reported that SARS-CoV-2 viral loads were closely associated with spike-specific IgA responses in the nasal samples of acute COVID-19 patients ${ }^{45}$. Since dlgA were shown to be about 15 times more potent than mlgA in vitro against the same target, dlgA were suggested to be particularly valuable for therapeutic application against SARS-CoV- ${ }^{46}$. It is essential to investigate the potential of mlgA and dlgA in preventing SARS-CoV-2 infection in vivo.

In this study, we used the technology of single B cell antibody gene cloning to generate a panel of SARSCoV-2 RBD-specific monoclonal HuNAbs from the peripheral blood mononuclear cells (PBMCs) of one acute and three convalescent COVID-19 patients in Hong Kong. Since intramuscular or intranasal inoculation of several potent IgG HuNAbs cannot completely prevent SARS-CoV-2 infection in the nasal turbinate (NT) of Syrian hamsters ${ }^{47}$, we sought to improve the efficacy of HuNAb by converting IgG to $\lg A$. To achieve this goal, we engineered the potent B8-IgG1 into monomeric $\lg A 1$ (B8-mlgA1), monomeric $\lg \mathrm{A} 2$ (B8-mlgA2), dimeric (B8-dlgA1) and dimeric $\lg \mathrm{A} 2$ (B8-dlgA2), and determined their efficacies in the Syrian hamster model against the live intranasal SARS-CoV-2 challenge ${ }^{48}$.

\section{Results}

\section{Characterization of human monoclonal antibodies from COVID-19 patients}


To isolate human monoclonal antibodies (MAbs), we obtained peripheral blood mononuclear cells (PBMCs) from one acute (P4) and three convalescent (P1-P3) COVID-19 patients in Hong Kong at a mean $73.5( \pm 25)$ days after symptoms onset (Supplementary Table 1). Enzyme-linked immunosorbent assay (ELISA) and pseudovirus neutralization assays revealed that all patient sera showed SARS-CoV-2 RBDand spike-specific binding (Fig. 1A and 1B) and neutralizing antibody (NAb) activities (Fig. 1C). The mean NAb IC ${ }_{50}$ titer was 1:1753 with a range of 1:638-1:5701. Flow cytometry was then used to sort SARS-CoV2-specific immunoglobulin positive ( $\mathrm{IgG}^{+}$) memory $B$ cells from individual PBMC samples using two fluorescent-conjugated RBD probes. The percentage of RBD-binding $\mathrm{IgG}^{+}$memory $\mathrm{B}$ cells ranged from $0.19 \%$ to $0.52 \%$ (Supplementary Fig. $1 \mathrm{~A}$ and Supplementary Fig. 1B). We successfully cloned a total of 34 MAbs from these patients, including 3 from P1, 8 from P2, 17 from P3 and 6 from P4. We confirmed that 18 of these MAbs exhibited RBD-specific binding activities detected by ELISA (Supplementary Fig. 1C). No clear dominance of heavy $(\mathrm{H})$ chain gene family was found among these 4 subjects by sequence analysis (Fig. 1D, left panels). VLK1, however, was the most used variable gene family for the light (L) chain (Fig. 1D, right panels). The average somatic hypermutation (SHM) rate ranged from $0 \%$ to $12.2 \%$ for the $\mathrm{H}$ chain and from $0.7 \%$ to $7.9 \%$ for the $\mathrm{L}$ chain (Fig. $1 \mathrm{E}$, left). The average complementaritydetermining region 3 (CDR3) lengths ranged from 12.3 to 17.4 for the $\mathrm{H}$ chain and 8.4 to 9.4 for the $\mathrm{L}$ chain, respectively (Fig. 1E, right). These results suggested overall comparable degrees of affinity maturation in these RBD-specific human MAbs obtained from individual memory B cells of four Hong Kong patients.

\section{Specificity and potency of SARS-CoV-2-specific human neutralizing antibodies}

To determine the antiviral activities of these 18 RBD-specific human MAbs, we performed binding and neutralization assays. Five of them, namely A6-IgG1, B4-lgG1, B7-IgG1, B8-IgG1 and C5-IgG1 displayed RBD- and spike-specific binding by ELISA (Fig. 1F) and neutralizing activities against both pseudotyped and authentic viruses (Fig. 1G and Supplementary Fig. 1D). Interestingly, the four most potent HuNAbs, B4-IgG1, B7-IgG1, B8-IgG1 and C5-IgG1, all came from patient P3 (Supplementary Fig. 1D). Sequence analysis revealed strong similarities between B7-, B8-, and C5-IgG1, which all contained an IGHV1-69 heavy chain gene and an IGKV3 kappa light chain gene. B4-IgG1 contained distinct IGHV3-66 and IGKV133 genes with CDR3 lengths of 12 amino acids (aa) and 9 aa, and somatic hypermutation (SHM) rate of $3.8 \%$ and $4.6 \%$, respectively (Supplementary Table 2). B7 and B8 were the most similar, as both contained IGHV1-69 and IGKV3-11 though B8 had a shorter CDR3 (14 aa vs 18 aa) and higher SHM (4.8\% vs $0.0 \%$ ) than those of $B 7$ in the heavy chain. In the $L$ chain, B7 and B8 shared a 9 aa CDR3, but B8 had a higher SHM rate than that of B7 (1.7\% vs $0.7 \%)$ C5-IgG1 had a similar IGHV1-69 light chain gene with a 16 aa CDR3 and $2.4 \%$ SHM, but a different IGKV3-20 with a 9 aa CDR3 and $2.8 \%$ SHM. By ELISA, these four P3-derived HuNAbs bound to the SARS-CoV-2 RBD with half-maximal effective concentration $\left(\mathrm{EC}_{50}\right)$ values ranging from 0.02 to $0.06 \mu \mathrm{g} / \mathrm{ml}$, indicating stronger binding than that of the P4-derived A6-lgG1 $(0.3 \mu \mathrm{g} / \mathrm{ml})$ (Fig. 1F, left and Supplementary Table 3). P3-derived MAbs also exhibited stronger binding 
activities to the spike, with the $\mathrm{EC}_{50}$ values ranging from 0.018 to $0.06 \mu \mathrm{g} / \mathrm{ml}$, compared to $\mathrm{A6}$ - $\mathrm{lg} \mathrm{G} 1$ $(17.94 \mu \mathrm{g} / \mathrm{ml})$ (Fig. 1F, right and Supplementary Table 3). Neutralizing assays using pseudoviruses revealed that these four potent HuNAbs had IC ${ }_{50}$ values ranging from 0.0095 to $0.038 \mu \mathrm{g} / \mathrm{ml}$, and IC $\mathrm{C}_{90}$ values ranging from 0.046 to $0.136 \mu \mathrm{g} / \mathrm{ml}$, respectively (Fig. 1G, left and Supplementary Table 4). Moreover, B8 proved to be the most potent HuNAb, capable of inhibiting authentic SARS-CoV-2 with an $\mathrm{IC}_{50}$ value of $0.013 \mu \mathrm{g} / \mathrm{ml}$ and an $\mathrm{IC}_{90}$ value of $0.032 \mu \mathrm{g} / \mathrm{ml}$, respectively (Fig. $1 \mathrm{G}$, right and

Supplementary Table 4). We then determined if these HuNAbs could compete with ACE2 for RBD binding by surface plasmon resonance (SPR). We found that all of them strongly competed with ACE2 (Fig. 1H). In line with the ELISA results, B8-IgG1 displayed the best KD value for RBD binding (169 pM) (Supplementary Fig. 2A, Supplementary Table 5) and the strongest competition with ACE2 (Fig. 1H), which explained its potent neutralizing activity. As B4-IgG1 displayed only partial competition for RBD binding with the other antibodies (Supplementary Fig. 2B), we further performed antibody synergy experiments using the pseudotype neuralization assay. No significant synergistic effects were found between any pairs of these four HuNAbs (Supplementary Fig. 2C). These results demonstrated that B4-, B7-, B8- and C5-IgG1 were all RBD-specific and competed with ACE2 for similar sites on the RBD.

\section{B8-IgG1 preexposure prophylaxis and post-exposure treatment in the golden Syrian hamster model}

To determine the efficiency of B8-IgG1 in pre-exposure prophylaxis and post-exposure treatment against live intranasal SARS-CoV-2 infection, we administered B8-IgG1 intraperitoneally in golden Syrian hamsters, before or after viral challenge in our Biosafety Level-3 (BSL-3) animal laboratory (Fig. 2A). In the pre-exposure prophylaxis group $(\mathrm{G} 1, \mathrm{n}=4)$, each hamster received a single intraperitoneal injection of $1.5 \mathrm{mg} / \mathrm{kg}$ B8-IgG1. In the post-exposure treatment groups, each hamster received a single intraperitoneal injection of $1.5 \mathrm{mg} / \mathrm{kg}$ B8-lgG1 at day $1(\mathrm{G} 2, \mathrm{n}=4)$, day $2(\mathrm{G} 3, \mathrm{n}=4)$ or day $3(\mathrm{G} 4, \mathrm{n}=4)$ postinfection (dpi), respectively (Fig. 2A). The challenge dose was $10^{5}$ plaque-forming units (PFU) of live SARS-CoV-2 (HKU-001a strain) ${ }^{47,48}$. Another group of hamsters $(G 0, n=4)$ received PBS injection as a no-treatment control. One G4 animal died accidentally during the procedure. Since Syrian hamsters recover quickly from SARS-CoV-2 infection, with resolution of clinical signs and clearance of virus shedding within one

week after infection 26,48 , we chose to sacrifice the animals at 4 dpi for HuNAb efficacy analysis, at a time when high viral loads and acute lung injury were consistently observed. At $4 \mathrm{dpi}$, NT and lung tissues were harvested to quantify infectious viruses by measuring PFUs, viral RNA loads by real-time reversetranscription polymerase chain reaction (RT-PCR) and infected cells by immunofluorescence (IF) staining of viral nucleocapsid protein (NP)-positive cells as we described previously 47,48 .

We found that infectious virus, measured by PFU, weas readily detected in all tissue compartments of G0 hamsters but not in the lungs of $75 \% \mathrm{G} 1,100 \% \mathrm{G} 2,75 \% \mathrm{G} 3$ and $0 \% \mathrm{G} 4$ animals, nor in the NT of $50 \% \mathrm{G} 1$, $75 \% \mathrm{G} 2,50 \% \mathrm{G} 3$ and $25 \% \mathrm{G} 4$ animals (Fig. 2B and 2E). The decrease in PFU was of 2-3 orders of 
magnitude, suggesting efficient viral suppression in the lungs for the G1, G2, and G3 groups. A sensitive RT-PCR assay further demonstrated that viral RNA copy numbers were decreased in the lungs by 3 orders of magnitude in $50 \%$ of $\mathrm{G} 1$ hamsters (Fig. $2 \mathrm{C}$ ). In contrast, there was no significant viral RNA reduction in the NT of G1 animals (Fig. 2F), suggesting lower efficacy of B8-IgG1 to prevent viral entry in the URT than in the lungs. There were slight but not significant viral RNA load decreases in both lungs and NT of G2 and some $\mathrm{G} 3$ animals. We then evaluated the number of infected cells or foci in these two tissue compartments by anti-NP antibody staining. A clear decrease of NP-positive cells or foci was observed in the lungs of $\mathrm{G} 1$ and $\mathrm{G} 2$ hamsters (Fig. 2D). Abundant NP-positive cells with a diffuse distribution, however, were readily detected in the NT of all the challenged hamsters (Fig. 2G). These results demonstrated that systemic B8-IgG1 injection was effective at reducing productive SARS-CoV-2 infection in the lungs when used for pre-exposure prophylaxis and early treatment especially within 48 hours post infection, but was insufficient to prevent viral infection in the NT.

To determine the correlates of B8-IgG1-mediated protection, we also measured the antibody concentration in serum, lung homogenate and NT homogenates at 0 and 4 dpi for all experimental animals. On average, $4,257 \mathrm{ng} / \mathrm{ml}$ and 2,101 ng/ml B8-IgG1 were found in animal sera at 0 and $4 \mathrm{dpi}$

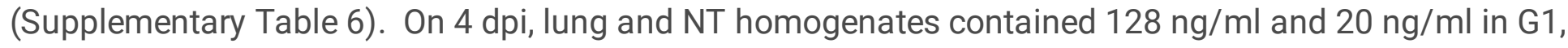
$238 \mathrm{ng} / \mathrm{ml}$ and $86 \mathrm{ng} / \mathrm{ml}$ in G2, $229 \mathrm{ng} / \mathrm{ml}$ and $93 \mathrm{ng} / \mathrm{ml}$ in G3, and $192 \mathrm{ng} / \mathrm{ml}$ and $46 \mathrm{ng} / \mathrm{ml}$ in G4 animals, respectively. These results demonstrated that most animals maintained higher peripheral B8IgG1 antibody concentration, while a decreasing concentration gradient was observed in the lungs and NT during infection. The concentrations of B8-IgG1 measured in NT homogenates were close to the neutralization $\mathrm{IC}_{90}$ measured in vitro $(32 \mathrm{ng} / \mathrm{ml})$, explaining why infectious virus was undetectable in the PFU assay. These findings are in line with results obtained for other potent IgG HuNAbs administered systemically, as reported in our previous study ${ }^{47}$. The B8-IgG1 concentrations measured in the NT appeared insufficient to completely block infection in vivo, as indicated by the presence of NP-positive cells scattered throughout the NT.

\section{Preexposure prophylaxis by monomeric B8-mlgA1 and B8-mlgA2 in Syrian hamsters}

Since systemic administration of the RBD-specific neutralizing B8-IgG1 did not suppress SARS-CoV-2 nasal infection effectively, we sought to construct various types of IgA for mucosal intervention. For this purpose, we engineered B8-IgG1 into monomeric B8-mlgA1 and B8-mlgA2, and then into dimeric B8-dlgA1 and $\mathrm{B} 8$-dlgA2 by introducing the $\mathrm{J}$ chain. By in vitro characterization, purified B8-mlgA1, B8-mlgA2, B8dlgA1 and B8-dlgA2 retained similar binding to RBD and spike by ELISA as compared to B8-lgG1 (Supplementary Fig. 3A-B, Supplementary Table 7), and comparable antiviral activities based on neutralization $\mathrm{IC}_{50}$ and $\mathrm{IC}_{90}$ values ((Supplementary Fig. $3 \mathrm{C}-\mathrm{D}$ and Supplementary Table 8). That said, B8-mlgA2 and B8-dlgA2 showed slightly more potent $\mathrm{IC}_{90}$ activities than B8-lgG1 in the pseudovirus 
neutralization assay using 293T-ACE2 cells as targets (Supplementary Table 8). After introducing $\mathrm{J}$ chain, the proper dimer formation of B8-dlgA1 and B8-dlgA2 was confirmed by size exclusion chromatography analysis (Supplementary Fig. 3E-F). Furthermore, B8-mlgA1, B8-mlgA2, B8-dlgA1 and B8-dlgA2 also retained comparable competition with ACE2 for binding to spike by SPR analysis (Supplementary Fig. 3GJ). Therefore, the engineered IgA had the expected structural properties, and showed antiviral activities as potent as those of B8-IgG1 in vitro.

We then evaluated the monomeric B8-mlgA1 and B8-mlgA2 in the hamster model, using a higher 4.5 $\mathrm{mg} / \mathrm{kg}$ dose via either intranasal or intraperitoneal injection, and used the same amount of intranasal B8IgG1 inoculation as a control (Fig. 3A to 3I). Interestingly, while changes in total RdRp and subgenomic sgNP viral RNA loads were not obvious (Fig. 3D to 3E), B8-IgG1 and B8-mlgA1 (both i.n. and i.p.), but not B8-mlgA2, were able to significantly suppress infectious virus production (PFU) in the lungs of $75 \%$ infected hamsters by 2 orders of magnitude (Fig. 3D). Sporadic infected cell foci were still detected in the lung sections by anti-NP staining (Fig. 4E and Supplementary Fig. 4A), suggesting that protection conferred by B8-IgG1 and B8-mIgA1 was not complete. B8-mlgA2 was not able to suppress viral RNA load nor PFU in the challenged hamsters, regardless of the route of antibody injection. On the other hand, like B8-lgG1, both B8-mlgA1 and B8-mlgA2 did not achieve significant viral suppression in the NT. After intranasal administration of either B8-mlgA1 or B8-mlgA2, some hamsters even showed a trend of slightly increased infectious virus production in NT, though this did not reach statistical significance (Fig. $3 \mathrm{~F}-3 \mathrm{H}$ ). Among these animals, the NP-positive cells were detected readily in the NT, as demonstrated by the whole section scanning, indicating the comparable distribution compared with the B8-IgG1 and notreatment groups (Fig. 3I). These results were consistent with many NP-positive cells observed in diffusely infected areas of NT by classic IF (Supplementary Fig. 4B). These results demonstrated that B8mlgA1 was more potent than B8-mlgA2 at limiting SARS-CoV-2 infection in the lungs, but that both mlgA did not prevent nor significantly limit SARS-CoV-2 nasal infection.

\section{B8-dlgA1 and B8-dlgA2 mediate enhancement of SARS-CoV-2 nasal infection and injury in Syrian hamsters}

Next, we tested the effects of dimeric B8-dlgA1 and B8-dlgA2 in Syrian hamsters. To improve protective efficacy of intranasal dlgA treatment, we included a 3-fold higher dosage group of $13.5 \mathrm{mg} / \mathrm{kg}$ besides the $4.5 \mathrm{mg} / \mathrm{kg}$ group (Fig. 4A to $4 \mathrm{I})$, and we shortened the interval between dlgA and virus inoculation to 12 hours (Fig. 4A). Both RdRp and sgNP viral RNA loads dropped significantly in the lungs of hamsters that received the high dose of B8-dlgA2 compared to the no-treatment group (Fig. 4B and 4C). Both B8dlgA1 and B8-dlgA2 at the high dose also suppressed infectious viruses (PFU) in the lungs of $75 \%$ and $100 \%$ treated hamsters, respectively (Fig. 4D). High dose B8-dlgA1 and B8-dlgA2 also decreased the number of NP-positive cells or foci in the lungs, with a more marked change for B8-dlgA2 (Fig. 4E and Supplementary Fig. 5A). Unexpectedly, however, we observed significantly enhanced SARS-CoV-2 nasal infection and tissue damage in most infected hamsters included the low and high dose groups of both 
B8-dlgA1 and B8-dlgA2, in all the four assays used (Fig. 4F to 4I). High dose administration of B8-dlgA1 or B8-dlgA2 resulted in increased PFU production in the NT by 37-fold and 81-fold, respectively, compared to the no-treatment group (Fig. 4H). Since our model showed comparable NT PFU on day 2 and day 4 as described previously ${ }^{48}$, this level of enhanced infection was unusual. It was also not observed with B8IgG1 or monomeric B8-mlgA1 and B8-mlgA2 treatment, as described above. Moreover, the distribution of NP-positive cells in hamsters treated with dimeric B8-IgA was broader and reached deeper into NT tissue compared to the no-treatment group, as shown by whole section scanning (Fig. $4 \mathrm{I}$ and Supplementary Fig. 5B), which was associated with more severe and extensive epithelium desquamation and luminal cell debris (Supplementary Fig. 5C). The density of nasal NP ${ }^{+}$cells was also significantly higher in B8-dlgA2treated hamsters than in PBS-treated animals (Supplementary Fig. 5D-E). It is therefore conceivable that treatment with dimeric B8-IgA expanded the type and distribution of target cells in the nasal epithelium. Critically, we confirmed that control dimeric dlgA1 and dlgA2 did not enhance SARS-CoV-2 infection under the same experimental conditions (Supplementary Fig. 6). These results demonstrated that, instead of inducing viral suppression, pre-exposure dimeric B8-dlgA1 and B8-dlgA2 enhanced SARS-CoV-2 nasal infection and injury significantly in Syrian hamsters, which was consistently found in three independent experiments.

To validate the role of B8-dlgA1 and B8-dlgA2, we then measured B8-lgA concentrations in the serum at day 0 and $4 \mathrm{dpi}$, and in the lung and NT homogenates at $4 \mathrm{dpi}$. B8-dlgA1 and B8-dlgA2 were primarily detected in lung homogenates at $4 \mathrm{dpi}$ and were apparently undetectable in the serum and NT homogenates (Supplementary Table 9). The enhanced viral replication in NT probably exhausted B8dlgA1 and B8-dlgA2 locally, through antibody-virus complex formation and clearance ${ }^{30}$. To address this possibility, we treated separately five groups of naïve Syrian hamsters ( $n=4$ per group) with each antibody at the $4.5 \mathrm{mg} / \mathrm{kg}$ dose. Twelve hours after the inoculation, antibody concentrations were readily detected in each tissues compartment (Supplementary Table 10). The highest concentrations of dlgA antibodies were found in lung homogenates, followed by nasal washes, NT homogenates, and serum. These results suggest that B8-dlgA1 and B8-dlgA2 concentrations in the NT and lungs were still above their neutralization $\mathrm{IC}_{90}$ values at the time of viral challenge, indicating that the results obtained in our experiments could not be explained by the insufficient amounts of antibodies.

\section{B8-dlgA1- and B8-dlgA2-mediated enhancement of SARS-CoV-2 infection via CD209}

Since B8 antibodies share the same binding site to the RBD domain, we sought to investigate possible mechanisms of B8-dlgA1- and B8-dlgA2-enhanced infection. First, we consistently found that $10 \mathrm{ng} / \mathrm{ml}$ B8-dlgA1 or B8-dlgA2 completely neutralized SARS-CoV-2 infection in human renal proximal tubule cells (HK-2), as measured in our previously reported immunofluorescence assay (Supplementary Fig. 7) ${ }^{49}$. We also tested B8-mlgA2 and B8-dlgA2 neutralizing capacity in the MucilAir ${ }^{\mathrm{TM}}$ model, consisting of a reconstructed human nasal epithelium, which contained goblet, ciliated, and basal cells (Fig. 5A) ${ }^{50}$. Both B8-mlgA2 and B8-dlgA2 neutralized SARS-CoV-2 in a dose-dependent fashion, when compared to a dlgA2 
control antibody. Similar experiments were carried out in the presence of the mucus naturally secreted by goblet cells, to determine whether dlgA interaction with the mucus may alter their neutralization capacity. However, B8-dlgA2 showed the same neutralization capacity in the presence and absence of mucus. These results demonstrated that B8-dlgA1 and B8-dlgA2 did not enhance SARS-CoV-2 infection in either human HK-2 or primary airway epithelial cells, which primarily expressed human ACE2 as a viral receptor. We then turned our attention to ACE2-independent mechanisms that might be associated with dimeric IgA-mediated enhancement of SARS-CoV-2 infection. Considering that mucosal monocyte-derived dendritic cells (DC) could mediate SARS-CoV-1 infection and dissemination in rhesus monkeys as early as $2 \mathrm{dpi}$, as we previously reported ${ }^{51}$, we sought to investigate the role of DC-expressed surface receptors. We focused on CD209 (DC-SIGN), because this lectin was previously shown to act as a cellular receptor for secretory $\lg A{ }^{52}$. By IF staining, intranasal administration of B8-dlgA2 alone did not increase CD209 expression in the NT of treated hamsters (Fig. 5B, left). Upon SARS-CoV-2 infection, however, we noted an increase in CD209-positive cells in olfactory epithelium devoid of ACE2 expression (Fig. 5B, middle). Importantly, most CD209 ${ }^{+}$cells were positive for NP (Fig. 5B, right), indicating that these CD209+ cells were likely permissive to SARS-CoV-2 infection. We then determined whether B8-dlgA1 and B8-dlgA2 could enhance SARS-CoV-2 infection in 293T cells expressing human CD209 or CD299 but not ACE2. Using a low MOI of 0.05, we found that pre-incubation of B8-dlgA1 and B8-dlgA2 enhanced live SARSCoV-2 infection significantly in 293T cells expressing human CD209, as determined by increased viral NP production (Fig. 5C). Interestingly, human CD299, a type II integral membrane protein that is $77 \%$ identical to CD209, did not show similar activities in the same experiment (Fig. 5C). Control dlgA1 and dlgA2 did not show any enhancement in $\mathrm{NP}^{+}$cell detection compared with virus only. Considering that CD209 ${ }^{+}$DCs promote HIV- 1 transmission to $\mathrm{CD}^{+}{ }^{+} \mathrm{T}$ cells via cell-cell contacts, we speculated that B8dlgA1 and B8-dlgA2 might not be able to block the similar process for SARS-CoV-2. Indeed, by testing the $\mathrm{B} 8$ antibodies at concentrations 100-times higher than $\mathrm{IC}_{90}$ neutralization values (around 3000 $\mathrm{ng} / \mathrm{ml}$ ), none of B8-lgG1, B8-mlgA1, B8-mlgA2, B8-dlgA1 and B8-dlgA2 could block cell-cell fusion (Fig. 5D). Taken together, our results demonstrated that B8-dlgA1- and B8-dlgA2-enhanced SARS-CoV-2 nasal infection likely involved viral capture and infection of mucosal $\mathrm{CD} 209^{+}$cells, followed by more robust infection of ACE2 ${ }^{+}$epithelial cells through trans-infection via cell-cell spread in NT.

\section{Cryo-EM analysis of the spike-B8 complex}

To understand the potential mechanism of action of B8 HuNAb, we performed a cryo-EM single-particle analysis of B8 Fab bound to the SARS-CoV-2 spike ectodomain trimer (Supplementary Fig. 8). Two B8spike complex structures were determined based on 351,095 and 616,799 particles collected, respectively (Supplementary Table 11). One structure at $2.67 \AA ̊$ resolution contained the spike with all three RBDs adopting the "up" conformation (3u), where each "up" RBD was bound by one B8 Fab (Fig. 6A-B). The other structure at $2.65 \AA$ resolution contained one spike trimer with 2 RBDs in the "up" conformation and 1 RBD in the "down" conformation (2u1d), where each RBD was also bound by one B8 Fab despite the 
presence of two distinct RBD conformations (Fig. 6C-D). After superimposing the "3u" and "2u1d" spikes, a 53-degree rotation was observed between the "up" RBD (red color) in the 3u spike trimer and the "down" RBD (gray) in the 2u1d spike trimer (Fig. 6E). The B8 Fab appeared to bind to the receptor-binding motif (RBM) of the RBD through its heavy chain for most of the interactions (Fig. 6F-G). Therefore, the cellular receptor ACE2 would clash with the B8 Fab due to the overlap of their respective epitopes on the RBM (Fig. 6F-H and Supplementary Table 12). The elucidation of the epitope revealed that B8 could be grouped into the SARS-CoV-2 neutralizing antibody class II ${ }^{53}$. These structural findings were further supported by neutralization assays using a panel of pseudoviruses containing naturally occurring mutations. Indeed, the E484K mutation from the South African SAD9 strain, which is located within the B8-binding interface, caused a major loss of neutralizing potency for all the B8 isotypes tested: IgG1, mlgA1, mlgA2, dlgA1 and dlgA2 (Supplementary Table 13). The comparable neutralization profiles of these NAbs against the full panel of viral variants also indicated that the conformation of key RBDbinding residues remained unchanged after engineering of the constant regions of these B8 isotypes.

\section{Discussion}

In this study, we investigated the preventive potential of a potent RBD-specific NAb B8 primarily in the forms of monomeric and dimeric IgA against live intranasal SARS-CoV-2 infection in the golden Syrian hamster model as compared with B8-IgG1 ${ }^{47,48}$. While these B8-IgA antibodies maintained neutralizing activities against SARS-CoV-2 in vitro similar to those of B8-lgG1, they displayed distinct in vivo effects, with clear differences in their capacity to modulate viral infection in the NT. Pretreatment by intranasal administration of $4.5 \mathrm{mg} / \mathrm{kg}$ of monomeric B8-mlgA1 or B8-mlgA2 did not significantly reduce infectious virus production in the NT homogenates. On the contrary, the antibody isotype had a marked effect, as intranasal administration of $4.5 \mathrm{mg} / \mathrm{kg}$ and $13.5 \mathrm{mg} / \mathrm{kg}$ dimeric B8-dlgA1 or B8-dlgA2 paradoxically increased the amount of infectious virus (PFU) in NT homogenates. This enhancing effect was not observed with several intranasal IgG HuNAbs previously tested by other groups or our team ${ }^{47,54}$. Mechanistically, instead of neutralization, virus-bound B8-dlgA1 and B8-dlgA2 used CD209 as an alternative receptor to infect non-ACE2 cells. CD209 ${ }^{+}$cells were increased and permissive to viral infection in the olfactory epithelium of Syrian hamsters upon SARS-CoV-2 infection, suggesting that this cell population could contribute to viral mucosal seeding. Indeed, we found that CD209 expressing cells could be infected in vitro by live SARS-CoV-2 at $0.05 \mathrm{MOI}$ in the presence of B8-dlgA1 and B8-dlgA2. Since none of the B8-based MAbs could prevent SARS-CoV-2 cell-to-cell transmission, even at high concentration in vitro, virus-laden mucosal CD209+ cells might trans-infect ACE2 ${ }^{+}$cells through cell-to-cell contacts in NT, resulting in enhanced infection and injury. Cryo-EM analysis further indicated that B8 is a typical class II HuNAb that binds to the SRAS-CoV-2 spike RBD in either a $3 u$ or a $2 u 1 d$ mode. Our findings, therefore, reveal a previously unrecognized pathway for RBD-specific dimeric IgA-mediated enhancement of SARS-CoV-2 nasal infection and injury in Syrian hamsters. 
The role of dimeric IgA has been explored primarily for mucosal transmitted viruses. At the mucosal surface, the major IgA type is the secretory form, which is generated from dlgA by the acquisition of a secretory component upon endocytosis and secretion by epithelial cells. In the simian AIDS macaque model, neutralizing dlgA given directly into the rectal lumen can prevent viral acquisition in rhesus monkeys challenged via the mucosal route ${ }^{55}$. Although the administered dlgA did not contain the secretory component (SC), they might have associated with free SC, which is present in mucosal secretions such as human lung lavages ${ }^{56}$. Neutralizing dlgA1 and dlgA2 could be protective through several mechanisms, including direct virus neutralization, virion capture, or the inhibition of virion transcytosis across the epithelium ${ }^{40}$. In this macaque study, however, Watkins et al. demonstrated that the dimeric HGN194 dlgA2 protected only $1 / 6$ animals in a rectal challenge model ${ }^{55}$. Recently, Taylor et al. found an increase in virion number and penetration depth in the transverse colon and mesenteric lymph nodes, after mucosal treatment with the HGN194 dlgA2 compared to a PBS control ${ }^{57}$. The authors suggested that virus-specific dlgA somehow mediated the delivery of virus immune complexes to the mesenteric lymph nodes for systemic infection. Here, we report that SARS-CoV-2 may subvert the action of potent neutralizing antibodies, as pretreatment with neutralizing B8-dlgA1 and B8-dlgA2 induced a more robust nasal infection via a previously unrecognized mode of viral enhancement.

SARS-CoV-2 engages CD209 ${ }^{+}$cells to evade ACE2-dependent neutralizing B8-dlgA1 and B8-dlgA2 for enhanced NT infection and injury. Previous studies have indicated various scenarios for ADE occurrence in viral infections. The well-known dengue $A D E$ has been associated with poorly neutralizing crossreactive antibodies against a heterologous viral serotype, leading to increased infection of FcyRexpressing cells ${ }^{58}$. Recent findings suggested that an increase in afucosylated antibodies contribute to dengue $A D E{ }^{59}$. In contrast, vaccine-associated enhanced respiratory disease induced by respiratory syncytial virus has not been found to be antibody-dependent ${ }^{60}$. For SARS and MERS, ADE observed in vitro depended on binding of the antibody Fab to the virus and the binding of the Fc component to FcyR on target cells ${ }^{61}$. One study found that spike IgG antibody abrogated wound-healing responses in SARSCoV-1-infected Chinese macaques ${ }^{62}$. In the case of COVID-19, vaccination and passive immunization studies have not revealed ADE of disease severity ${ }^{63}$. Comprehensive studies, however, are necessary to define the clinical correlates of protective immunity against SARS-CoV-2, especially in the context of vaccine breakthrough infections. During natural infection, one study indicated that the increase in afucosylated antibodies might contribute to COVID-19 severity ${ }^{64}$. To date, four classes of potent HuNAbs have been isolated from convalescent COVID-19 patients ${ }^{34,53}$. The molecular mechanism of neutralization for most potent HuNAbs was primarily through blocking the interaction between ACE2 and the spike RBD. Currently, systemic RBD-specific HuNAb treatment remains to be improved for therapeutic suppression of SARS-CoV-2 replication in the NT or URT, both in animal models and human trials $47,48,65$. One limitation is the insufficient amounts of HuNAbs distributed on the nasal mucosal surface for protection ${ }^{47}$. Other reasons might include alternative entry pathways engaged by SARS-CoV-2 
to evade HuNAbs. To this end, Liu et al. reported recently that antibodies against the spike N-terminal domain (NTD) induced an open conformation of the RBD and thus enhanced the binding capacity of the spike to the ACE2 receptor, leading to increased viral infectivity ${ }^{66}$. Yeung et al. demonstrated nicely that SARS-CoV-2 could engage soluble ACE2 (SACE2) and then bind alternate receptors for viral entry, through interaction between a spike/sACE2 complex with the angiotensin II AT1 receptor, or interaction between a spike/sACE2/vasopressin complex with the AVPR1B vasopressin receptor, respectively ${ }^{49}$. In this study, we found that, in the presence of potent neutralizing B8-dlgA1 or B8-dlgA2 antibodies, SARS-CoV-2 used the cellular receptor CD209 for capture or infection, which likely expanded the use of $C D 209^{+}$cells as target cells, leading to enhanced NT infection and trans-infection. Interestingly, a preprint report suggests that cells expressing CD209 can be infected directly by SARS-CoV-2 through an interaction of the spike with the NTD instead of the RBD ${ }^{67}$. This mode of action, however, was unlikely to explain our findings, because no enhancement of SARS-CoV-2 nasal infection was found in presence of control dlgA1 and dlgA2. Our results rather suggest that the direct binding of virus-bound B8-dlgA1 or virus-bound B8-dlgA2 to CD209 is a likely pathway, resulting in the more severe SARS-CoV-2 nasal infection and damage. In line with our results, a previous study demonstrated that dlgA itself can use CD209 as a cellular receptor ${ }^{52}$. During the entry process, since neither B8-dlgA1 nor B8-dlgA2 could prevent virus cell-to-cell transmission, infected mucosal $\mathrm{CD} 209^{+}$cells might enable a more robust viral transmission to ciliated nasal epithelial cells in NT, which show the highest expression of ACE2 and TMPRSS2 receptors ${ }^{65}$. In support of this notion, previous studies indicated that mucosal DCs can capture HIV- 1 through binding of its envelope glycoproteins to CD209 and efficiently transfer the bound virions to CD ${ }^{+} \mathrm{T}$ cells, in a process called trans-enhancement or trans-infection ${ }^{68}$. The trans-infection markedly decreased the neutralization efficiency of potent NAbs directed at HIV ${ }^{69}$. Moreover, although monocyte-derived DCs (MDDCs) cannot support productive SARS-CoV-2 replication ${ }^{70}$, a recent study demonstrated that MDDCs could mediate efficient viral trans-infection of the Calu-3 human respiratory cell line ${ }^{71}$. Our findings of increased number of infectious viruses in NT, therefore, have significant implications for SARS-CoV-2 transmission, COVID19 pathogenesis, and immune interventions.

\section{Limitations of the study}

As our in vivo findings were obtained in Syrian hamsters, it remains to be determined whether CD209+ DCs are abundantly recruited to the nasal mucosa in SARS-CoV-2-infected humans. It is, however, known that myeloid DCs are increased in the nasal epithelium upon infection ${ }^{51,72}$. Our preliminary analysis of the human nasal cytology data (under accession code EGAS00001004082) revealed the presence of increased CD209+ DCs in addition to abundant ACE2, TMPRSS2, and furin expression in the apical side of multiciliated cells of SARS-CoV-2-infected human subjects (Supplementary Fig. 9) ${ }^{73}$. Another limitation is that we did not have an NTD-specific neutralizing dlgA for comparison. Besides the class II neutralizing dlgA such as B8-mlgA2 and B8-dlgA2 used in this study, neutralizing dlgA belonging to other classes should also be investigated in future. We also do not know whether other cellular receptor such 
as the polymeric immunoglobulin receptor (plgR) plays a role in B8-dlgA-enhanced SARS-CoV-2 nasal infection. The plgR is responsible for transcytosis of soluble dlgAs and immune complexes from the basolateral to the apical epithelial surface. It remains uncertain whether B8-dlgA-enhanced NT infection would lead to worse neuro-COVID-19. Because current intramuscular vaccinations might not induce secretory dlgA at the nasal mucosal sites ${ }^{74}$, it remains unknown whether the dlgA-mediated ADE would happen in people who received the COVID-19 vaccines or dlgA treatment. Future studies are needed to address these limitations.

\section{Methods}

\section{Human subjects}

A total of 4 patients with COVID-19 including 3 convalescent cases and one acute case were recruited between February and May 2020. All patients were confirmed by reverse-transcription polymerase chain reaction (RT-PCR) as described previously ${ }^{28}$. Clinical and laboratory findings were entered into a predesigned database. Written informed consent was obtained from all patients. This study was approved by the Institutional Review Board of The University of Hong Kong/Hospital Authority Hong Kong West Cluster, the Hong Kong East Cluster Research Ethics Committee, and the Kowloon West Cluster Research Ethics Committee (UW 13-265, HKECREC-2018-068, KW/EX-20-038[144-26]).

\section{Syrian hamsters}

The animal experimental plan was approved by the Committee on the Use of Live Animals in Teaching and Research (CULATR 5359-20) of the University of Hong Kong (HKU). Male and female golden Syrian hamsters (Mesocricetus auratus) (aged 6-10 weeks) were purchased from the Chinese University of Hong Kong Laboratory Animal Service Centre through the HKU Laboratory Animal Unit (LAU). The animals were kept in Biosafety Level-2 housing and given access to standard pellet feed and water ad libitum following LAU's standard operational procedures (SOPS). The viral challenge experiments were then conducted in our Biosafety Level-3 animal facility following SOPs strictly, with strict adherence to SOPs

\section{Cell lines}

HEK293T cells, HEK293T-hACE2 cells Vero-E6 cells, HK2 cells and Vero-E6-TMPRSS2 cells were maintained in DMEM containing 10\% FBS, $2 \mathrm{mM} \mathrm{L}$-glutamine, $100 \mathrm{U} / \mathrm{mL} / \mathrm{mL}$ penicillin and incubated at $37^{\circ} \mathrm{C}$ in a $5 \% \mathrm{CO}_{2}$ setting ${ }^{62}$. Expi293F ${ }^{\mathrm{TM}}$ cells were cultured in Expi293TM Expression Medium (Thermo Fisher Scientific) at $37{ }^{\circ} \mathrm{C}$ in an incubator with $80 \%$ relative humidity and a $5 \% \mathrm{CO}_{2}$ setting on an orbital 
shaker platform at $125 \pm 5 \mathrm{rpm} / \mathrm{min}$ (New Brunswick innova ${ }^{\mathrm{TM}} 2100$ ) according to the manufacturer's instructions.

\section{ELISA analysis of plasma and antibody binding to RBD and trimeric spike}

The recombinant RBD and trimeric spike proteins derived from SARS-CoV-2 (Sino Biological) were diluted to final concentrations of $1 \mu \mathrm{g} / \mathrm{mL} / \mathrm{mL}$, then coated onto 96-well plates (Corning 3690) and incubated at $4{ }^{\circ} \mathrm{C}$ overnight. Plates were washed with PBS-T (PBS containing $0.05 \%$ Tween-20) and blocked with blocking buffer (PBS containing $5 \%$ skim milk or $1 \% \mathrm{BSA}$ ) at $37^{\circ} \mathrm{C}$ for $1 \mathrm{~h}$. Serially diluted plasma samples or isolated monoclonal antibodies were added to the plates and incubated at $37^{\circ} \mathrm{C}$ for $1 \mathrm{~h}$. Wells were then incubated with a secondary goat anti-human $\lg G$ labelled with horseradish peroxidase (HRP) (Invitrogen) or with a rabbit polyclonal anti-human IgA alpha-chain labelled with HRP (Abcam) and TMB substrate (SIGMA). Optical density (OD) at $450 \mathrm{~nm}$ was measured by a spectrophotometer. Serially diluted plasma from healthy individuals or previously published monoclonal antibodies against HIV-1 (VRC01) were used as negative controls.

\section{Isolation of RBD-specific lgG+ single memory B cells by FACS}

RBD-specific single B cells were sorted as previously described ${ }^{75}$. In brief, PBMCs from infected individuals were collected and incubated with an antibody cocktail and a His-tagged RBD protein for identification of RBD-specific B cells. The cocktail consisted of the Zombie viability dye (Biolegend), CD19-Percp-Cy5.5, CD3-Pacific Blue, CD14-Pacific Blue, CD56-Pacific Blue, IgM-Pacific Blue, IgD- Pacific Blue , IgG-PE, CD27-PE-Cy7 (BD Biosciences) and the recombinant RBD-His described above. Two consecutive staining steps were conducted: the first one used an antibody and RBD cocktail incubation of $30 \mathrm{~min}$ at $4{ }^{\circ} \mathrm{C}$; the second staining involved staining with anti-His-APC and anti-His-FITC antibodies (Abcam) at $4{ }^{\circ} \mathrm{C}$ for 30 min to detect the His tag of the RBD. The stained cells were washed and resuspended in PBS containing $2 \%$ FBS before being strained through a 70- $\mu \mathrm{m}$ cell mesh filter (BD Biosciences). RBD-specific single $B$ cells were gated as $\mathrm{CD}^{2} 19^{+} \mathrm{CD} 27^{+} \mathrm{CD} 3^{-} \mathrm{CD} 14^{-} \mathrm{CD} 56^{-} \mathrm{lgM} \mathrm{M}^{-} \mathrm{lg} \mathrm{D}^{-} \mathrm{lg} \mathrm{G}^{+} \mathrm{RBD}^{+}$ and sorted into 96-well PCR plates containing $10 \mu \mathrm{L}$ of RNAase-inhibiting RT-PCR catch buffer (1M Tris$\mathrm{HCl} \mathrm{pH} \mathrm{8.0,} \mathrm{RNase} \mathrm{inhibitor,} \mathrm{DEPC-treated} \mathrm{water).} \mathrm{Plates} \mathrm{were} \mathrm{then} \mathrm{snap-frozen} \mathrm{on} \mathrm{dry} \mathrm{ice} \mathrm{and} \mathrm{stored} \mathrm{at}$ $-80^{\circ} \mathrm{C}$ until the reverse transcription reaction.

\section{Single B cell RT-PCR and antibody cloning}

Single memory B cells isolated from PBMCs of infected patients were cloned as previously described ${ }^{76}$. Briefly, one-step RT-PCR was performed on sorted single memory B cell with a gene specific primer mix, followed by nested PCR amplifications and sequencing using the heavy chain and light chain specific 
primers. Cloning PCR was then performed using heavy chain and light chain specific primers containing specific restriction enzyme cutting sites (heavy chain, 5'-Agel/3'-Sall; kappa chain, 5'-Agel/3'-BsiWI). The PCR products were purified and cloned into the backbone of antibody expression vectors containing the constant regions of human Igy 1 or Iga1 and Iga2. The Iga1 and Iga2 vectors were purchased from InvivoGen (pfusess-hcha1 for IgA1 and pfusess-hcha2m1 for IgA2). The constructed plasmids containing paired heavy and light chain expression cassettes were co-transfected into 293T cells (ATCC) grown in 6well plates. Antigen-specific ELISA and pseudovirus-based neutralization assays were used to analyze the binding capacity to SARS-CoV-2 RBD and the neutralization capacity of transfected culture supernatants, respectively.

\section{Genetic analysis of the BCR repertoire}

Heavy chain and light chain germline assignment, framework region annotation, determination of somatic hypermutation (SHM) levels (in nucleotides) and CDR loop lengths (in amino acids) were performed with the aid of the IMGT/HighV-QUEST software tool suite (www.imgt.org/HighV-QUEST). Sequences were aligned using Clustal W in the BioEdit sequence analysis package (Version 7.2). Antibody clonotypes were defined as a set of sequences that share genetic $\mathrm{V}$ and $\mathrm{J}$ regions as well as an identical CDR3.

\section{Antibody production and purification}

The paired antibody VH/VL chains were cloned into Igy, Iga1 or lga2 and Ig expression vectors using T4 ligase (NEB). For production of $\lg G$ and monomeric $\lg A$, the plasmids with paired heavy chain (IgG, $\lg A 1$, IgA2) and light chain genes were co-transfected into Expi293 ${ }^{\mathrm{TM}}$ expression system (Thermo Fisher Scientific) following the manufacturer's protocol to produce recombinant monoclonal antibodies. For dlgA antibody production, plasmids of paired heavy chain (IgA1, IgA2) and kappa light chain together with a J chain were co-transfected into Expi293 ${ }^{\mathrm{TM}}$ expression system (Thermo Fisher Scientific) at the ratio of 1:1:1 following the manufacturer's instructions. Antibodies produced from cell culture supernatants were purified immediately by affinity chromatography using recombinant Protein G-Agarose (Thermo Fisher Scientific) or CaptureSelect ${ }^{\mathrm{TM}} \mathrm{IgA}$ Affinity Matrix (Thermo Fisher Scientific) according to the manufacturer's instructions, to purify IgG and IgA, respectively. The purified antibodies were concentrated by an Amicon ultracentrifuge filter device (molecular weight cut-off $10 \mathrm{kDa}$; Millipore) to a volume of $0.2 \mathrm{~mL}$ in PBS (Life Technologies), and then stored at $4{ }^{\circ} \mathrm{C}$ or $-80^{\circ} \mathrm{C}$ for further characterization.

\section{Size exclusion chromatography}


The prepacked HiLoad 26/60 Superdex ${ }^{\mathrm{TM}}$ 200pg (code No. 17-1071-01, Cytiva) column was installed onto the Amersham Biosciences AKTA FPLC system. After column equilibration with 2 column volumes (CV) of PBS, the concentrated IgA antibodies were applied onto the column using a 500-ul loop at a flow rate of $2 \mathrm{~mL} / \mathrm{min}$. Dimers of $\mathrm{IgA} 1$ or $\mathrm{IgA} 2$ were separated from monomers upon washing with $2 \mathrm{CV}$ of PBS. The milli-absorbance unit at OD280nm was recorded during the washing process. $2 \mathrm{~mL}$-fractions were collected, pooled, concentrated and evaluated by western blot using mouse anti-IGJ monoclonal antibody [KT109] (Abcam) and rabbit anti-human IgA alpha chain antibody (Abcam).

\section{Pseudovirus-based neutralization assay}

The neutralizing activity of NAbs was determined using a pseudotype-based neutralization assay as we previously described ${ }^{77}$. Briefly, The pseudovirus was generated by co-transfection of 293T cells with pVax-1-S-COVID19 and pNL4-3Luc_Env_Vpr, carrying the optimized spike (S) gene (QHR63250) and a human immunodeficiency virus type 1 backbone, respectively ${ }^{77}$. Viral supernatant was collected at $48 \mathrm{~h}$ post-transfection and frozen at $-80^{\circ} \mathrm{C}$ until use. The serially diluted monoclonal antibodies or sera were incubated with $200 \mathrm{TCID}_{50}$ of pseudovirus at $37^{\circ} \mathrm{C}$ for 1 hour. The antibody-virus mixtures were subsequently added to pre-seeded HEK 293T-ACE2 cells. 48 hours later, infected cells were lysed to measure luciferase activity using a commercial kit (Promega, Madison, WI). Half-maximal $\left(\mathrm{IC}_{50}\right)$ or $90 \%$ $\left(\mathrm{IC}_{90}\right)$ inhibitory concentrations of the evaluated antibody were determined by inhibitor vs. normalized response -4 Variable slope using GraphPad Prism 6 or later (GraphPad Software Inc.).

\section{Neutralization activity of monoclonal antibodies against authentic SARS-CoV-2}

The SARS-CoV-2 focus reduction neutralization test (FRNT) was performed in a certified Biosafety level 3 laboratory. Neutralization assays against live SARS-CoV-2 were conducted using a clinical isolate (HKU001a strain, GenBank accession no: MT230904.1) previously obtained from a nasopharyngeal swab from an infected patient ${ }^{78}$. The tested antibodies were serially diluted, mixed with $50 \mu \mathrm{L}$ of SARS-CoV-2 $\left(1 \times 10^{3}\right.$ focus forming unit $\left./ \mathrm{mL}, \mathrm{FFU} / \mathrm{mL}\right)$ in 96 -well plates, and incubated for 1 hour at $37^{\circ} \mathrm{C}$. Mixtures were then transferred to 96 -well plates pre-seeded with $1 \times 10^{4} /$ well Vero E6 cells and incubated at $37^{\circ} \mathrm{C}$ for 24 hours. The culture medium was then removed and the plates were air-dried in a biosafety cabinet (BSC) for 20 mins. Cells were then fixed with a $4 \%$ paraformaldehyde solution for 30 min and air-dried in the BSC again. Cells were further permeabilized with $0.2 \%$ Triton X-100 and incubated with cross-reactive rabbit sera anti-SARS-CoV-2-N for 1 hour at RT before adding an Alexa Fluor 488 goat anti-rabbit lgG $(\mathrm{H}+\mathrm{L})$ cross-adsorbed secondary antibody (Life Technologies). The fluorescence density of SARS-CoV-2 infected cells were scanned using a Sapphire Biomolecular Imager (Azure Biosystems) and the neutralization effects were then quantified using Fiji software $(\mathrm{NIH})$. 


\section{Antibody binding kinetics, and competition with the ACE2 receptor measured by Surface Plamon Resonance (SPR)}

The binding kinetics and affinity of recombinant monoclonal antibodies for the SARS-CoV-2 spike protein (ACROBiosystems) were analysed by SPR (Biacore 8K, GE Healthcare). Specifically, the spike protein was covalently immobilized to a CM5 sensor chip via amine groups in $10 \mathrm{mM}$ sodium acetate buffer $(\mathrm{pH} 5.0)$ for a final RU around 500. SPR assays were run at a flow rate of $30 \mathrm{~mL} / \mathrm{min}$ in HEPES buffer. For conventional kinetic/dose-response, serial dilutions of monoclonal antibodies were injected across the spike protein surface for 180 s, followed by a 600 s dissociation phase using a multi-cycle method. Remaining analytes were removed in the surface regeneration step with the injection of $10 \mathrm{mM}$ glycine$\mathrm{HCl}(\mathrm{pH} 2.0)$ for $2 \times 30 \mathrm{~s}$ at a flow rate of $30 \mu \mathrm{l} / \mathrm{min}$. Kinetic analysis of each reference subtracted injection series was performed using the Biacore Insight Evaluation Software (GE Healthcare). All sensorgram series were fit to a 1:1 (Langmuir) binding model of interaction. Before evaluating the competition between antibodies and the human ACE2 peptidase domain, both the saturating binding concentrations of antibodies and of the ACE2 protein (ACROBiosystems) for the immobilized SARS-CoV-2 spike protein were determined separately. In the competitive assay, antibodies at the saturating concentration were injected onto the chip with immobilized spike protein for 120 s until binding steady-state was reached. ACE2 protein also used at the saturating concentration was then injected for 120 s, followed by another 120 s of injection of antibody to ensure a saturation of the binding reaction against the immobilized spike protein. The differences in response units between ACE2 injection alone and prior antibody incubation reflect the antibodies' competitive ability against ACE2 binding to the spike protein.

\section{Hamster experiments}

In vivo evaluation of monoclonal antibody B8-IgG1, B8-mlgA1, B8-mlgA2, B8-dlgA1, B8-dlgA2 in the established golden Syrian hamster model of SARS-CoV-2 infection was performed as described previously, with slight modifications ${ }^{48}$. Approval was obtained from the University of Hong Kong (HKU) Committee on the Use of Live Animals in Teaching and Research. Briefly, 6-8-week-old male and female hamsters were housing with access to standard pellet feed and water ad libitum until live virus challenge in the BSL-3 animal facility. The hamsters were randomized from different litters into experimental groups. Experiments were performed in compliance with the relevant ethical regulations ${ }^{48}$. For prophylaxis studies, 24 hours before live virus challenge, three groups of hamsters were intraperitoneally or intranasally administered with one dose of test antibody in phosphate-buffered saline (PBS) at the indicated dose. At day 0 , each hamster was intranasally inoculated with a challenge dose of $100 \mu \mathrm{L}$ of Dulbecco's Modified Eagle Medium containing $10^{5}$ PFU of SARS-CoV-2 (HKU-001a strain, GenBank accession no: MT230904.1) under anaesthesia with intraperitoneal ketamine $(200 \mathrm{mg} / \mathrm{kg})$ and xylazine $(10 \mathrm{mg} / \mathrm{kg})$. For pre-treatment study, each hamster received one $1.5 \mathrm{mg} / \mathrm{kg}$ dose of intraperitoneal B8- 
IgG1 at $24,48,72$ hours ( $n=4$ per group) after virus challenge. The hamsters were monitored twice daily for clinical signs of disease. Syrian hamsters typically clear virus within one week after SARS-CoV-2 infection. Accordingly, animals were sacrificed for analysis at day 4 after virus challenge with high viral loads ${ }^{48}$. Half the nasal turbinate, trachea, and lung tissues were used for viral load determination by quantitative SARS-CoV-2-specific RdRp/Hel RT-qPCR assay ${ }^{28}$ and infectious virus titration by plaque assay ${ }^{48}$.

\section{Cryo-EM sample preparation and data acquisition}

The purified SARS-CoV-2 S-B8 protein complexes were concentrated before being applied to the grids. Aliquots $(4 \mu \mathrm{L})$ of the protein complex were placed on glow-discharged holey carbon grids (Quantifoil $\mathrm{Au}$ $\mathrm{R} 1.2 / 1.3,300$ mesh). The grids were blotted and flash-frozen in liquid ethane cooled by liquid nitrogen with a Vitrobot apparatus (Mark IV, ThermoFisher Scientific). The grids sample quality was verified with an FEl Talos Arctica 200-kV electron microscope (Thermo Fisher Scientific). The verified grids with optimal ice thickness and particle density were transferred to a Titan Krios operating at $300 \mathrm{kV}$ and equipped with a Cs corrector, a Gatan K3 Summit detector (Gatan Inc.) and a GIF Quantum energy filter (slit width $20 \mathrm{eV}$ ). Micrographs were recorded in the super-resolution mode with a calibrated pixel size of $0.54895 \AA$ A. Each movie has a total accumulated exposure of 50 e-/Å2 fractionated in 32 frames. The final image was binned 2 -fold to a pixel size of $1.0979 \AA$. AutoEMation was used for the fully automated data collection. The defocus value of each image, which was set from -1.0 to $-2.0 \mu \mathrm{m}$ during data collection, was determined by Gctf. Data collection statistics are summarized in Supplementary Table 11.

\section{Cryo-EM data processing}

The procedure for image processing of SARS-CoV-2 S-B8 complex is presented in Supplementary Fig. 2. In brief, Motion Correction (MotionCo2), CTF-estimation (GCTF) and non-templated particle picking (Gautomatch, http://www.mrc-Imb.cam.ac.uk/kzhang/) were automatically executed by the TsinghuaTitan.py program (developed by Dr. fang Yang). Sequential data processing was carried out on RELION 3.0 and RELION 3.1. Initially, 2,436,776 particles were auto-picked by Gautomatch or RELION 3.0 from 4213 micrographs. After several 2D classifications, 1,451,176 particles were selected and applied for 3D classification with one class. Two different states were obtained after further 3D classification: 3 RBD in up conformation bound with B8 Fab (3u), and 2 up RBDs and 1 down RBD with each bound to a B8 Fab (2u1d). 616,799 particles for the 2u1d state and 351,095 particles for the $3 u$ state were subjected to 3D auto-refinement, yielding final resolutions at $3.21 \AA$ and $3.06 \AA$, respectively. Further CTF refinement and Bayesian polishing improved the resolution to $2.65 \AA$ ( $2 \mathrm{u} 1 \mathrm{~d}, \mathrm{C} 1$ symmetry) and $2.67 \AA$ ( $3 \mathrm{u}, \mathrm{C} 3$ symmetry) with better map quality. To improve the RBD-B8 portion map density, focused local search classification was applied for each RBD-B8 portion with an adapted soft mask. The best classes for each RBD-Fab portion were selected and yielded a final resolution at $3.56 \AA$ (RBD-Fab1, up), $3.34 \AA$ (RBD-Fab2, 
up), $3.69 \AA$ (RBD-Fab3, down), $3.87 \AA$ (RBD-Fab3, up) from 479,305, 508,653, 656,429, and 136,482 particles, respectively. Further CTF refinement and Bayesian polishing improved the resolution of RBDFab2 to 3.11 A with better map quality. RBD-Fab maps were fitted onto the whole structure map using Chimera, then combined using PHENIX combine_focused_maps. The reported resolutions were estimated with the gold-standard Fourier shell correlation (FSC) cutoff of 0.143 criterion. Data processing statistics are summarized in Supplementary Table 11.

\section{Model building and structure refinement}

The spike model (PDB code: 6VSB) and the initial model of the B8 Fab generated by SWISS-Model were fitted into the EM density map, and further manually adjusted with Coot. Glocusides were built manually with carbohydrate tool in Coot. The atomic models were refinement using Phenix in real space with secondary structure and geometry restraints. The final structures were validated using Phenix.molprobity. UCSF Chimera, ChimeraX and PyMol were used for map segmentation and figure generation. Model refinement statistics are summarized in Supplementary Table 11.

\section{SARS-CoV-2 infection of reconstructed human nasal epithelia}

MucilAir ${ }^{T M}$, corresponding to reconstructed human nasal epithelium cultures differentiated in vitro for at least 4 weeks, were purchased from Epithelix (Saint-Julien-en-Genevois, France). The cultures were generated from pooled nasal tissues obtained from 14 human adult donors. Cultures were maintained in air/liquid interface (ALI) conditions in transwells with $700 \mu \mathrm{L}$ of MucilAir ${ }^{\mathrm{TM}}$ medium (Epithelix) in the basal compartment, and then kept at $37^{\circ} \mathrm{C}$ under a 5\% CO2 atmosphere. SARS-CoV-2 infection was performed as previously described ${ }^{50}$. Briefly, the apical side of ALI cultures was washed 20 min at $37^{\circ} \mathrm{C}$ in Mucilair ${ }^{\mathrm{TM}}$ medium to remove mucus. Cells were then incubated with $10^{4}$ plaque-forming units (PFU) of the isolate BetaCoV/France/IDF00372/2020 (EVAg collection, Ref-SKU: 014V-03890; kindly provided by S. Van der Werf). The viral input was diluted in DMEM medium to a final volume $100 \mu \mathrm{L}$, and then left on the apical side for $4 \mathrm{~h}$ at $37^{\circ} \mathrm{C}$. Control wells were mock treated with DMEM medium (Gibco) for the same duration. Viral inputs were removed by washing twice with $200 \mu \mathrm{L}$ of PBS ( 5 min at $37^{\circ} \mathrm{C}$ ) and once with $200 \mu \mathrm{L}$ Mucilair ${ }^{\mathrm{TM}}$ medium $\left(20 \mathrm{~min}\right.$ at $\left.37^{\circ} \mathrm{C}\right)$. The basal medium was replaced every 2-3 days. Apical supernatants were harvested every 2-3 days by adding $200 \mu \mathrm{L}$ of Mucilair ${ }^{\mathrm{TM}}$ medium on the apical side, with an incubation of $20 \mathrm{~min}$ at $37^{\circ} \mathrm{C}$ prior to collection. For IgA treatment, cultures were washed once and then pretreated with antibodies added to the apical compartment for $1 \mathrm{~h}$ in $50 \mu \mathrm{L}$. Viral input was then directly added to reach a final volume of $100 \mu \mathrm{L}$. The antibodies were added again at day 2 d.p.i. in the apical compartment during an apical wash $\left(20 \mathrm{~min}\right.$ at $\left.37^{\circ} \mathrm{C}\right)$. To test the effect of dlgA treatment in the presence of mucus, dlgA were added directly to the apical compartment of MucilAir ${ }^{\mathrm{TM}}$ cultures without an initial wash. After IgA treatment for $1 \mathrm{~h}$, the virus was added directly to the $\lg A /$ mucus mixture and left on 
the apical side for $4 \mathrm{~h}$ at $37^{\circ} \mathrm{C}$. After viral inoculation, a single brief wash was made to remove the viral input while limiting mucus loss. The cultures were then maintained as in the no-mucus condition.

\section{Viral RNA quantification in reconstructed human nasal epithelia}

Apical supernatants were collected, stored at $-80^{\circ} \mathrm{C}$ until thawing and were then diluted 4 -fold in PBS in a 96-well plate. Diluted supernatants were inactivated for $20 \mathrm{~min}$ at $80^{\circ} \mathrm{C}$. For SARS-CoV-2 RNA quantification, $1 \mu \mathrm{L}$ of diluted supernatant was added to $4 \mu \mathrm{L}$ of PCR reaction mix. PCR was carried out in 384-well plates using the Luna Universal Probe One-Step RT-qPCR Kit (New England Biolabs) with SARSCoV-2 NP-specific primers (Forward 5'-TAA TCA GAC AAG GAA CTG ATT A-3'; Reverse 5'-CGA AGG TGT GAC TTC CAT G-3') on a QuantStudio 6 Flex thermocycler (Applied Biosystems). A standard curve was established in parallel using purified SARS-CoV-2 viral RNA.

\section{Histopathology and immunofluorescence (IF) staining}

The lung and nasal turbinate tissues collected at necropsy were fixed in zinc formalin and then processed into paraffin-embedded tissue blocks. The tissue sections $(4 \mu \mathrm{m})$ were stained with hematoxylin and eosin (H\&E) for light microscopy examination as previously described with modifications ${ }^{47}$. For identification and localization of SARS-CoV-2 nucleocapsid protein (NP) in organ tissues, immunofluorescence staining was performed on deparaffinized and rehydrated tissue sections using a rabbit anti-SARS-CoV-2-NP protein antibody together with an AF488-conjugated anti-rabbit IgG (Jackson ImmunoResearch, PA, USA). Briefly, the tissue sections were first treated with antigen unmasking solution (Vector Laboratories) in a pressure cooker. After blocking with $0.1 \%$ Sudan black B for 15 min and $1 \%$ bovine serum albumin (BSA)/PBS at RT for 30 min, the primary rabbit anti-SARS-CoV-2-NP antibody (1:4000 dilution with $1 \% \mathrm{BSA} / \mathrm{PBS}$ ) was incubated at $4^{\circ} \mathrm{C}$ overnight. This step was followed by incubation with a FITC-conjugated donkey anti-rabbit IgG (Jackson ImmunoResearch) for $30 \mathrm{~min}$ and the sections were then mounted in medium with 4',6-diamidino-2-phenylindole (DAPI). For identification of DC-SIGN expression, we stained the NT slices with rabbit anti-DC-SIGN primary antibody (Abcam) and Alexa Fluor 488 goat anti-rabbit IgG $(\mathrm{H}+\mathrm{L})$ cross-adsorbed secondary antibody (Life Technologies) according to the manufacturer's instructions. For identification of ACE2 expression, the goat anti-ACE2 primary antibody (R\&D) and Alexa Fluor 568 donkey anti-goat $\lg G(\mathrm{H}+\mathrm{L})$ secondary antibodies (Invitrogen) according to the manufacturer's instructions. All tissue sections were examined, and the fluorescence images and whole section scanning were captured using $5 \times, 10 \times$ and $20 \times$ objectives with Carl Zeiss LSM 980. NP ${ }^{+}$cells per field were quantified based on the mean fluorescence intensity (MFI) using the ZEN BLACK 3.0 and Image $(\mathrm{NIH})$. 


\section{Effects of B8-dlgA on SARS-CoV-2 infection in HK2 cells}

HK2 cells were seeded into 24-well plates at the 40-50\% confluency and cultured overnight. The B8-dlgA or control dlgA at the concentration of $1,10,100,1000 \mathrm{ng} / \mathrm{ml} / \mathrm{mL}$ and then mixed with SARS-CoV-2 (1:10 TCID50) and incubated for 1 hour at room temperature. The antibody/virus mixture was then added to HK2 cells after the cell culture medium was removed and washed with PBS once and incubated for 1 hour at $37^{\circ} \mathrm{C}$. The infectious medium was replaced with fresh medium containing respective concentration of antibody after washing 3 times with PBS. $24 \mathrm{~h}$ later, the infected cells were imaged under fluorescence microscope after staining with AF488-conjugated anti-SARS-CoV-2 NP antibody. Alternatively, the infected cells were lysed and blotted for SARS-CoV-2 NP protein to determine the extent of infection. Tubulin was blotted as the internal control.

\section{B8-dlgA mediated enhancement via CD209}

HEK293T cells were seeded into $10-\mathrm{cm}$ dish at $40 \%$ confluency and cultured overnight. The HEK293T cells were transfected with human CD209 (Sino Biological) at 70\%-90\% confluency. The expression of CD209 was measured by flow cytometry. The transfected HEK293T-CD209 cells were seeded into 96-well plates with $2.4 \times 10^{4}$ cells per well and cultured overnight. The HEK293T-CD209 cells were pre-treated with $10 \mathrm{ng} / \mathrm{ml} / \mathrm{mL}$ of B8-dlgA or control dlgA and incubated for $6 \mathrm{~h}$ prior SARS-CoV-2 infection (MOI=0.05). 24 $\mathrm{h}$ later, cells were then fixed with $4 \%$ paraformaldehyde solution for $30 \mathrm{~min}$ and air-dried in the BSC. Cells were further permeabilized with $0.2 \%$ Triton $\mathrm{X}-100$ and incubated with cross-reactive rabbit sera antiSARS-CoV-2-N for 1 hour at RT before adding Alexa Fluor 488 goat anti-rabbit lgG $(\mathrm{H}+\mathrm{L})$ cross-adsorbed secondary antibody (Life Technologies). The fluorescence density of SARS-CoV-2 infected cells was acquired using a Sapphire Biomolecular Imager (Azure Biosystems) and then the MFI of four randomly selected areas of each sample was quantified using Fiji software $(\mathrm{NIH})$.

\section{Effects of B8 antibodies on SARS-CoV-2 mediated cell-cell fusion}

Vero-E6 TMPRSS2 cells were seeded into 48-well plates and cultured overnight. After treatment with B8 antibodies at the dose of $3000 \mathrm{ng} / \mathrm{ml} / \mathrm{mL}$ for 1 hour, HEK293T cells transfected with SARS-CoV-2 spikeGFP were added into the treated Vero-E6 TMPRSS2 cells and co-cultured for 48 hours. The cell-cell fusion between Vero-E6 TMPRSS2 and HEK293T-Spike-GFP was then determined under a fluorescence microscope (Nikon ELIPSE) and the images of randomly selected region were captured using $4 \times$ and $10 \times$ objectives using the Nikon software.

\section{Re-analysis of published nasal brushing single-cell data}


The preprocessed scRNA-seq data from nasal brushing samples of 2 healthy controls and 4 COVID-19 patients were downloaded from Gene Expression Omnibus (GEO) database with accession numbers GSE171488 and GSE164547. Quality control metrics were consistent with the original article [PMID: 34003804] and performed based on the R package Seurat (version 4.0.3) [PMID: 34062119]. Harmony [PMID: 31740819] was used to integrate the samples based on the top 4000 most variable genes obtained with the FindVariableFeatures $\left(\right.$ function in Seurat. $\mathrm{CD}_{14}{ }^{+}$(monocyte) cells were extracted for further analysis. The annotation of the cell type was performed by manually checking the marker genes of each cluster identified from the FindAllMarkers() function in Seurat.

\section{Quantification and statistical analysis}

Statistical analysis was performed using PRISM 6.0 or later. Ordinary one-way ANOVA and multiple comparisons were used to compare group means and differences between multiple groups. Unpaired Student's $t$ tests were used to compare group means between two groups only. A P-value $<0.05$ was considered significant. The the number of independent experiments performed, the number of animals in each group, and the specific details of statistical tests are reported in the figure legends and the Methods section.

\section{Declarations}

\section{SUPPLEMENTAL INFORMATION}

The supplemental information includes 13 Tables and 9 Figures.

\section{ACKNOWLEDGMENTS}

We acknowledge financial supports from the Hong Kong Research Grants Council Collaborative Research Fund (C7156-20G to Z.C.); the National Key Research and Development Project of China (2020YFC0860600) and the National Program on Key Research Project of China (2020YFA0707500 and 2020YFA0707504); the Health and Medical Research Fund (COVID1903010-7), the Food and Health Bureau, The Government of the Hong Kong Special Administrative Region; Innovation and Technology Fund (ITF), The Government of the Hong Kong Special Administrative Region; the University Development Fund and Li Ka Shing Faculty of Medicine Matching Fund from the University of Hong Kong to the AIDS Institute; the Consultancy Service for Enhancing Laboratory Surveillance of Emerging Infectious Diseases and Research Capability on Antimicrobial Resistance for Department of Health of the Hong Kong Special Administrative Region Government; Sanming Project of Medicine in Shenzhen, China (SZSM201911014); the High Level-Hospital Program, Health Commission of Guangdong Province, China; the research project of Hainan academician innovation platform (YSPTZX202004); and the Hainan talent development 
project (SRC200003); L.A.C's team was supported by the Urgence COVID-19 Fundraising Campaign of Institute Pasteur (TROPICORO project). The study was also supported by generous donations of the Friends of Hope Education Fund, Lee Wan Keung Charity Foundation Limited, Shaw Foundation Hong Kong, Michael Seak-Kan Tong, Richard Yu and Carol Yu, May Tam Mak Mei Yin, Hong Kong Sanatorium \& Hospital, Hui Ming, Hui Hoy and Chow Sin Lan Charity Fund Limited, Chan Yin Chuen Memorial Charitable Foundation, Marina Man-Wai Lee, the Hong Kong Hainan Commercial Association South China Microbiology Research Fund, the Jessie \& George Ho Charitable Foundation, Perfect Shape Medical Limited, Kai Chong Tong, Tse Kam Ming Laurence, Foo Oi Foundation Limited, Betty Hing-Chu Lee, Ping Cham So, and Lo Ying Shek Chi Wai Foundation. Z.C.'s team was also partly supported by the Theme-Based Research Scheme (T11-706/18-N to Z.C.).

\section{AUTHOR CONTRIBUTIONS}

Conceptualization, Z.C.; HuNAb cloning, Z.B.; experimental design, Z.C., Z.B., R.Z., J.F.W.C.; hamster experiments, J.F.W.C., C.C.S.C., V.K.M.P., C.C.Y.C., K.K.H.C., and JC; cryoEM study, J.Z., J.G., Z.Y.W., X.W.; SPR experiments, Q.Z., S.S. and L.Z.; LL confocal imaging, L.L. and D.Z.; MucilAir ${ }^{\mathrm{TM}}$ experiment, R.R. and L.A.C.; HK-2 experiment, M.L.Y.; nasal cytology data analysis, M.Y. and R.S.; clinical specimens, K.K.W.T.; in vitro experiments, H.C., Z.D., K.K.A., H.H., H.O.M., J.C., C.L., J.Z.; manuscript preparation, Z.C., Z.B., R.Z., L.Z., K.Y.Y.; study supervision, Z.C., K.Y.Y. and L.Z.

\section{DECLARATION OF INTEREST}

J.F.W.C. has received travel grants from Pfizer Corporation Hong Kong and Astellas Pharma Hong Kong Corporation Limited and was an invited speaker for Gilead Sciences Hong Kong Limited and Luminex Corporation. The funding sources had no role in study design, data collection, analysis or interpretation or writing of the report. The other authors declare no conflicts of interest except for a provisional patent application filed for human monoclonal antibodies generated in our laboratory.

\section{References}

1. Zhu, N. et al. A Novel Coronavirus from Patients with Pneumonia in China, 2019. N Engl J Med 382, 727-733, doi:10.1056/NEJMoa2001017 (2020).

2. Zhou, P. et al. A pneumonia outbreak associated with a new coronavirus of probable bat origin. Nature 579, 270-273, doi:10.1038/s41586-020-2012-7 (2020).

3. Wan, Y., Shang, J., Graham, R., Baric, R. S. \& Li, F. Receptor recognition by novel coronavirus from Wuhan: An analysis based on decade-long structural studies of SARS. J Viro/ 94, e00127-00120, doi:10.1128/JVI.00127-20 (2020). 
4. Chan, J. F. et al. Genomic characterization of the 2019 novel human-pathogenic coronavirus isolated from a patient with atypical pneumonia after visiting Wuhan. Emerg Microbes Infect 9, 221-236, doi:10.1080/22221751.2020.1719902 (2020).

5. Lu, G., Wang, Q. \& Gao, G. F. Bat-to-human: spike features determining 'host jump' of coronaviruses SARS-CoV, MERS-CoV, and beyond. Trends Microbio/ 23, 468-478, doi:10.1016/j.tim.2015.06.003 (2015).

6. Shang, J. et al. Cell entry mechanisms of SARS-CoV-2. Proc Natl Acad Sci U S A 117, 11727-11734, doi:10.1073/pnas.2003138117 (2020).

7. Belouzard, S., Millet, J. K., Licitra, B. N. \& Whittaker, G. R. Mechanisms of coronavirus cell entry mediated by the viral spike protein. Viruses 4, 1011-1033, doi:10.3390/v4061011 (2012).

8. Wang, Q. et al. Structural and Functional Basis of SARS-CoV-2 Entry by Using Human ACE2. Cel/ 181, 894-904 e899, doi:10.1016/j.cell.2020.03.045 (2020).

9. Hoffmann, M. et al. SARS-CoV-2 Cell Entry Depends on ACE2 and TMPRSS2 and Is Blocked by a Clinically Proven Protease Inhibitor. Cell 181, 271-280 e278, doi:10.1016/j.cell.2020.02.052 (2020).

10. Lan, J. et al. Structure of the SARS-CoV-2 spike receptor-binding domain bound to the ACE2 receptor. Nature 581, 215-220, doi:10.1038/s41586-020-2180-5 (2020).

11. Walls, A. C. et al. Structure, Function, and Antigenicity of the SARS-CoV-2 Spike Glycoprotein. Cell 181, 281-292 e286, doi:10.1016/j.cell.2020.02.058 (2020).

12. Yan, R. et al. Structural basis for the recognition of SARS-CoV-2 by full-length human ACE2. Science 367, 1444-1448, doi:10.1126/science.abb2762 (2020).

13. Lu, R. et al. Genomic characterisation and epidemiology of 2019 novel coronavirus: implications for virus origins and receptor binding. Lancet 395, 565-574, doi:10.1016/S0140-6736(20)30251-8 (2020).

14. Wu, F. et al. A new coronavirus associated with human respiratory disease in China. Nature 579, 265-269, doi:10.1038/s41586-020-2008-3 (2020).

15. WHO. Weekly epidemiological update on COVID-19-27 July 2021, https://www.who.int/publications/m/item/weekly-epidemiological-update-on-covid-19--27-july-2021, 2021).

16. Cheng, V. C., Lau, S. K., Woo, P. C. \& Yuen, K. Y. Severe acute respiratory syndrome coronavirus as an agent of emerging and reemerging infection. Clin Microbiol Rev 20, 660-694, doi:10.1128/CMR.00023-07 (2007).

17. Rothe, C. et al. Transmission of 2019-nCoV Infection from an Asymptomatic Contact in Germany. $N$ Engl J Med 382, 970-971, doi:10.1056/NEJMc2001468 (2020).

18. Holshue, M. L. et al. First Case of 2019 Novel Coronavirus in the United States. N Engl J Med 382, 929-936, doi:10.1056/NEJMoa2001191 (2020).

19. Chan, J. F. et al. A familial cluster of pneumonia associated with the 2019 novel coronavirus indicating person-to-person transmission: a study of a family cluster. Lancet 395, 514-523, 
doi:10.1016/S0140-6736(20)30154-9 (2020).

20. Wang, D. et al. Clinical Characteristics of 138 Hospitalized Patients With 2019 Novel CoronavirusInfected Pneumonia in Wuhan, China. JAMA 323, 1061-1069, doi:10.1001/jama.2020.1585 (2020).

21. Johnson, B. A. et al. Loss of furin cleavage site attenuates SARS-CoV-2 pathogenesis. Nature 591, 293-299, doi:10.1038/s41586-021-03237-4 (2021).

22. Wong, Y. C. et al. Natural transmission of bat-like SARS-CoV-2PRRA variants in COVID-19 patients. Clin Infect Dis 73, e437-e444, doi:10.1093/cid/ciaa953 (2020).

23. Chu, H. et al. Host and viral determinants for efficient SARS-CoV-2 infection of the human lung. Nat Commun 12, 134, doi:10.1038/s41467-020-20457-w (2021).

24. Gobeil, S. M. et al. D614G Mutation Alters SARS-CoV-2 Spike Conformation and Enhances Protease Cleavage at the S1/S2 Junction. Cell Rep 34, 108630, doi:10.1016/j.celrep.2020.108630 (2021).

25. Korber, B. et al. Tracking Changes in SARS-CoV-2 Spike: Evidence that D614G Increases Infectivity of the COVID-19 Virus. Cell 182, 812-827 e819, doi:10.1016/j.cell.2020.06.043 (2020).

26. Plante, J. A. et al. Spike mutation D614G alters SARS-CoV-2 fitness. Nature 592, 116-121, doi:10.1038/s41586-020-2895-3 (2020).

27. Hung, I. F. et al. SARS-CoV-2 shedding and seroconversion among passengers quarantined after disembarking a cruise ship: a case series. Lancet Infect Dis 20, 1051-1060, doi:10.1016/S14733099(20)30364-9 (2020).

28. Chan, J. F. et al. Improved Molecular Diagnosis of COVID-19 by the Novel, Highly Sensitive and Specific COVID-19-RdRp/Hel Real-Time Reverse Transcription-PCR Assay Validated In Vitro and with Clinical Specimens. J Clin Microbio/ 58, e00310-00320, doi:10.1128/JCM.00310-20 (2020).

29. To, K. K. et al. Temporal profiles of viral load in posterior oropharyngeal saliva samples and serum antibody responses during infection by SARS-CoV-2: an observational cohort study. Lancet Infect Dis 20, 565-574, doi:10.1016/S1473-3099(20)30196-1 (2020).

30. Arvin, A. M. et al. A perspective on potential antibody-dependent enhancement of SARS-CoV-2. Nature 584, 353-363, doi:10.1038/s41586-020-2538-8 (2020).

31. Shi, R. et al. A human neutralizing antibody targets the receptor-binding site of SARS-CoV-2. Nature 584, 120-124, doi:10.1038/s41586-020-2381-y (2020).

32. Zost, S. J. et al. Potently neutralizing and protective human antibodies against SARS-CoV-2. Nature 584, 443-449, doi:10.1038/s41586-020-2548-6 (2020).

33. Liu, L. et al. Potent neutralizing antibodies against multiple epitopes on SARS-CoV-2 spike. Nature 584, 450-456, doi:10.1038/s41586-020-2571-7 (2020).

34. Cao, Y. et al. Potent Neutralizing Antibodies against SARS-CoV-2 Identified by High-Throughput Single-Cell Sequencing of Convalescent Patients' B Cells. Cell 182, 73-84 e16, doi:10.1016/j.cell.2020.05.025 (2020).

35. Robbiani, D. F. et al. Convergent antibody responses to SARS-CoV-2 in convalescent individuals. Nature 584, 437-442, doi:10.1038/s41586-020-2456-9 (2020). 
36. Sun, Z. et al. Potent neutralization of SARS-CoV-2 by human antibody heavy-chain variable domains isolated from a large library with a new stable scaffold. MAbs 12, 1778435, doi:10.1080/19420862.2020.1778435 (2020).

37. Wu, Y. et al. A noncompeting pair of human neutralizing antibodies block COVID-19 virus binding to its receptor ACE2. Science 368, 1274-1278, doi:10.1126/science.abc2241 (2020).

38. Wu, Y. et al. Identification of Human Single-Domain Antibodies against SARS-CoV-2. Cell Host Microbe 27, 891-898 e895, doi:10.1016/j.chom.2020.04.023 (2020).

39. Pilette, C., Ouadrhiri, Y., Godding, V., Vaerman, J. P. \& Sibille, Y. Lung mucosal immunity: immunoglobulin-A revisited. Eur Respir J 18, 571-588, doi:10.1183/09031936.01.00228801 (2001).

40. Corthesy, B. Multi-faceted functions of secretory IgA at mucosal surfaces. Front Immunol 4, 185, doi:10.3389/fımmu.2013.00185 (2013).

41. Ejemel, M. et al. A cross-reactive human IgA monoclonal antibody blocks SARS-CoV-2 spike-ACE2 interaction. Nat Commun 11, 4198, doi:10.1038/s41467-020-18058-8 (2020).

42. Sterlin, D. et al. IgA dominates the early neutralizing antibody response to SARS-CoV-2. Sci Trans/ Med 13, eabd2223, doi:10.1126/scitranslmed.abd2223 (2021).

43. Drummer, H. E. et al. Dimeric IgA is a specific biomarker of recent SARS-CoV-2 infection. medRxiv, 2021.2006.2028.21259671, doi:10.1101/2021.06.28.21259671 (2021).

44. Dos Santos, J. M. B. et al. In Nasal Mucosal Secretions, Distinct IFN and IgA Responses Are Found in Severe and Mild SARS-CoV-2 Infection. Front Immuno/ 12, 595343, doi:10.3389/fimmu.2021.595343 (2021).

45. Smith, N. et al. Distinct systemic and mucosal immune responses during acute SARS-CoV-2 infection. Nat Immunol, doi:10.1038/s41590-021-01028-7 (2021).

46. Wang, Z. et al. Enhanced SARS-CoV-2 neutralization by dimeric IgA. Sci Trans/ Med 13, eabf1555, doi:10.1126/scitranslmed.abf1555 (2021).

47. Zhou, D. et al. Robust SARS-CoV-2 infection in nasal turbinates after treatment with systemic neutralizing antibodies. Cell Host Microbe 29, 551-563, doi:10.1016/j.chom.2021.02.019 (2021).

48. Chan, J. F.-W. et al. Simulation of the clinical and pathological manifestations of Coronavirus Disease 2019 (COVID-19) in golden Syrian hamster model: implications for disease pathogenesis and transmissibility. Clinical Infectious Diseases 71, 2428-2446, doi:10.1093/cid/ciaa325 (2020).

49. Yeung, M. L. et al. Soluble ACE2-mediated cell entry of SARS-CoV-2 via interaction with proteins related to the renin-angiotensin system. Cell 184, 2212-2228 e2212, doi:10.1016/j.cell.2021.02.053 (2021).

50. Robinot, R. et al. SARS-CoV-2 infection induces the dedifferentiation of multiciliated cells and impairs mucociliary clearance. Nat Commun 12, 4354, doi:10.1038/s41467-021-24521-x (2021).

51. Liu, L. et al. Spatiotemporal interplay of severe acute respiratory syndrome coronavirus and respiratory mucosal cells drives viral dissemination in rhesus macaques. Mucosal Immuno/ 9, 10891101, doi:10.1038/mi.2015.127 (2016). 
52. Baumann, J., Park, C. G. \& Mantis, N. J. Recognition of secretory IgA by DC-SIGN: implications for immune surveillance in the intestine. Immunol Lett 131, 59-66, doi:10.1016/j.imlet.2010.03.005 (2010).

53. Wang, R. et al. Analysis of SARS-CoV-2 variant mutations reveals neutralization escape mechanisms and the ability to use ACE2 receptors from additional species. Immunity 54, 1611-1621 e1615, doi:10.1016/j.immuni.2021.06.003 (2021).

54. Yang, L. et al. COVID-19 antibody therapeutics tracker: a global online database of antibody therapeutics for the prevention and treatment of COVID-19. Antib Ther 3, 205-212, doi:10.1093/abt/tbaa020 (2020).

55. Watkins, J. D. et al. Anti-HIV IgA isotypes: differential virion capture and inhibition of transcytosis are linked to prevention of mucosal R5 SHIV transmission. AIDS 27, F13-20, doi:10.1097/QAD.0b013e328360eac6 (2013).

56. Merrill, W. W. et al. Free secretory component and other proteins in human lung lavage. Am Rev Respir Dis 122, 156-161, doi:10.1164/arrd.1980.122.1.156 (1980).

57. Taylor, R. A. et al. PET/CT targeted tissue sampling reveals virus specific dlgA can alter the distribution and localization of HIV after rectal exposure. PLoS Pathog 17, e1009632, doi:10.1371/journal.ppat.1009632 (2021).

58. Beltramello, M. et al. The Human Immune Response to Dengue Virus Is Dominated by Highly CrossReactive Antibodies Endowed with Neutralizing and Enhancing Activity. Cell Host \& Microbe 8, 271283, doi:10.1016/j.chom.2010.08.007 (2010).

59. Bournazos, S. et al. Antibody fucosylation predicts disease severity in secondary dengue infection. Science 372, 1102-1105, doi:10.1126/science.abc7303 (2021).

60. van Erp, E. A., Luytjes, W., Ferwerda, G. \& van Kasteren, P. B. Fc-Mediated Antibody Effector Functions During Respiratory Syncytial Virus Infection and Disease. Front Immunol 10, 548, doi:10.3389/fimmu.2019.00548 (2019).

61. Wan, Y. et al. Molecular Mechanism for Antibody-Dependent Enhancement of Coronavirus Entry. $J$ Virol 94, e02015-02019, doi:10.1128/JVI.02015-19 (2020).

62. Liu, L. et al. Anti-spike IgG causes severe acute lung injury by skewing macrophage responses during acute SARS-CoV infection. JCI Insight 4, e123158, doi:10.1172/jci.insight.123158 (2019).

63. Haynes, B. F. et al. Prospects for a safe COVID-19 vaccine. Sci Trans/ Med 12, doi:10.1126/scitranslmed.abe0948 (2020).

64. Larsen, M. D. et al. Afucosylated IgG characterizes enveloped viral responses and correlates with COVID-19 severity. Science 371, doi:10.1126/science.abc8378 (2021).

65. Baum, A. et al. REGN-COV2 antibodies prevent and treat SARS-CoV-2 infection in rhesus macaques and hamsters. Science 370, 1110-1115, doi:10.1126/science.abe2402 (2020).

66. Liu, Y. et al. An infectivity-enhancing site on the SARS-CoV-2 spike protein targeted by antibodies. Cell 184, 3452-3466 e3418, doi:10.1016/j.cell.2021.05.032 (2021). 
67. Soh, W. T. et al. The N-terminal domain of spike glycoprotein mediates SARS-CoV-2 infection by associating with L-SIGN and DC-SIGN. bioRxiv, 2020.2011.2005.369264, doi:10.1101/2020.11.05.369264 (2020).

68. Geijtenbeek, T. B. H. et al. DC-SIGN, a dendritic cell-specific HIV-1-binding protein that enhances transinfection of T cells. Cell 100, 587-597, doi:Doi 10.1016/S0092-8674(00)80694-7 (2000).

69. Bracq, L., Xie, M., Benichou, S. \& Bouchet, J. Mechanisms for Cell-to-Cell Transmission of HIV-1. Front Immuno/ 9, 260, doi:10.3389/fimmu.2018.00260 (2018).

70. Yang, D. et al. Attenuated Interferon and Proinflammatory Response in SARS-CoV-2-Infected Human Dendritic Cells Is Associated With Viral Antagonism of STAT1 Phosphorylation. J Infect Dis 222, 734-745, doi:10.1093/infdis/jiaa356 (2020).

71. Thepaut, M. et al. DC/L-SIGN recognition of spike glycoprotein promotes SARS-CoV-2 trans-infection and can be inhibited by a glycomimetic antagonist. PLoS Pathog 17, e1009576, doi:10.1371/journal.ppat.1009576 (2021).

72. Hartmann, E. et al. Analysis of plasmacytoid and myeloid dendritic cells in nasal epithelium. Clin Vaccine Immunol 13, 1278-1286, doi:10.1128/CVI.00172-06 (2006).

73. Ahn, J. H. et al. Nasal ciliated cells are primary targets for SARS-CoV-2 replication in the early stage of COVID-19. J Clin Invest 131, e148517, doi:10.1172/JCl148517 (2021).

74. Krammer, F. SARS-CoV-2 vaccines in development. Nature 586, 516-527, doi:10.1038/s41586-0202798-3 (2020).

75. Kong, L. et al. Key gp120 Glycans Pose Roadblocks to the Rapid Development of VRC01-Class Antibodies in an HIV-1-Infected Chinese Donor. Immunity 44, 939-950, doi:10.1016/j.immuni.2016.03.006 (2016).

76. Smith, K. et al. Rapid generation of fully human monoclonal antibodies specific to a vaccinating antigen. Nat Protoc 4, 372-384, doi:10.1038/nprot.2009.3 (2009).

77. Poeran, J., Zhong, H., Wilson, L., Liu, J. \& Memtsoudis, S. G. Cancellation of elective surgery and intensive care unit capacity in New York state: a retrospective cohort analysis. Anesth Analg 131, 1337-1341, doi:10.1213/ANE.0000000000005083 (2020).

78. Chu, H. et al. Comparative tropism, replication kinetics, and cell damage profiling of SARS-CoV-2 and SARS-CoV with implications for clinical manifestations, transmissibility, and laboratory studies of COVID-19: an observational study. The Lancet Microbe 1, e14-e23, doi:10.1016/S26665247(20)30004-5 (2020).

\section{Figures}


Fig. 1

A

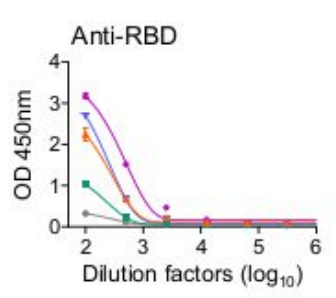

B

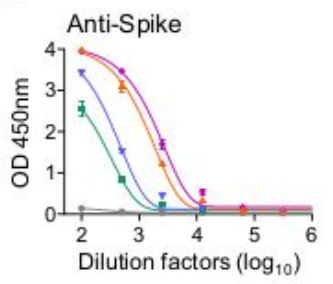

C

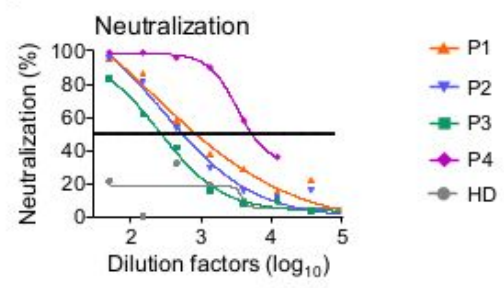

D
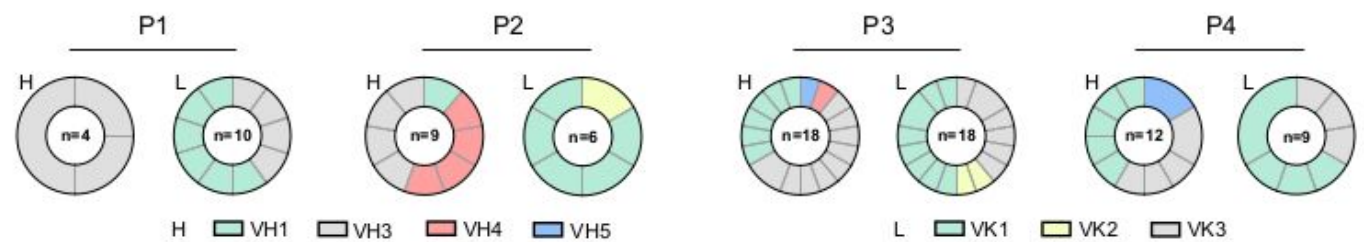

$\mathrm{H} \square \mathrm{VH} 1$

SHM CDR3 Length

L $\square$ VK1 $\square$ VK2 $\square$ VK3
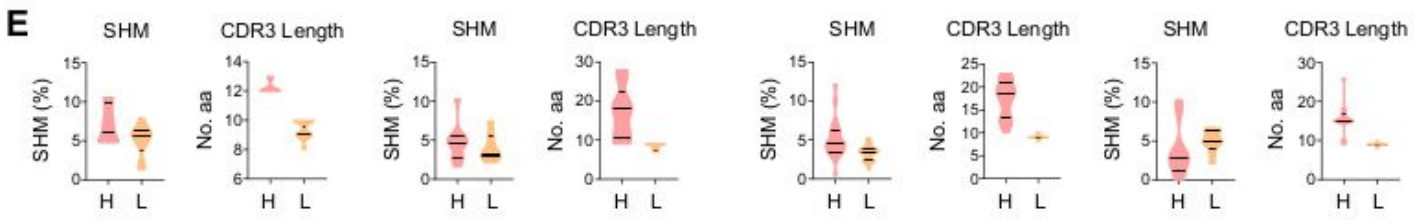

F
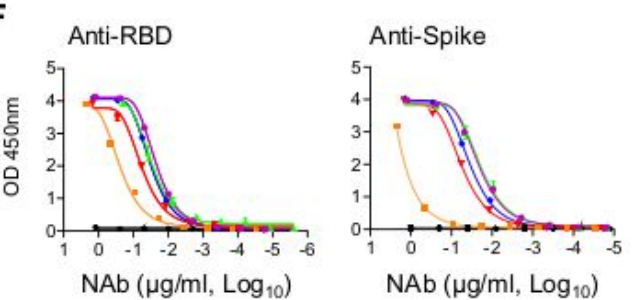

G
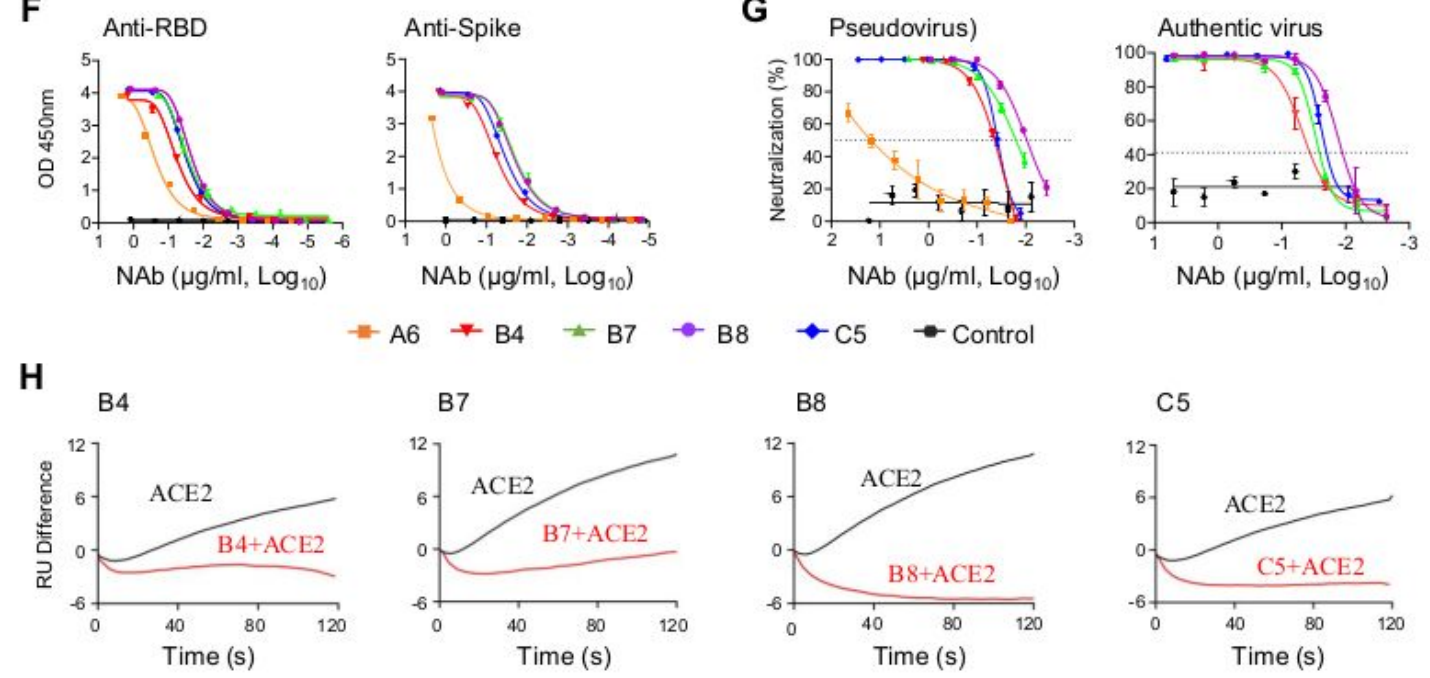

$\rightarrow \mathrm{A} 6 \rightarrow \mathrm{B} 4 \rightarrow \mathrm{B} 7 \rightarrow \mathrm{B} 8$
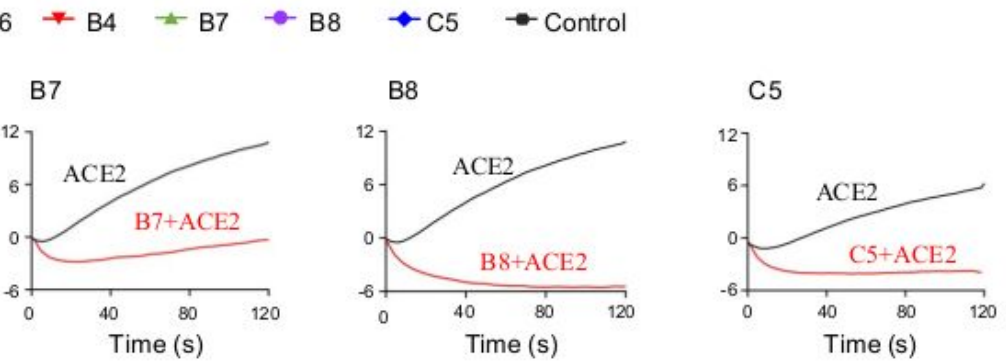

\section{Figure 1}

Isolation of monoclonal antibodies from single B cells of convalescent COVID-19 patients. (A) RBDspecific binding activities of sera derived from 3 (P1-P3) convalescent and 1 (P4) acute COVID-19 patients as measured by ELISA. (B) Spike-specific binding activities of sera derived from four COVID-19 patients as measured by ELISA. (C) Neutralization activities of sera derived from four COVID-19 patients as measured by pseudotyped SARS-CoV-2 inhibition in 293T-ACE2 cells. (D) Antibody gene repertoire 
analysis of reactive $B$ cells derived from each patient. The number of cloned antibody genes from each patient is shown in the center of each pie chart for both the heavy $(H)$ and light $(L)$ chains. The colors represent specific variable gene family. Each fragment of the same color stands for one specific subfamily. (E). The percentage of somatic hypermutation (SHM) compared to germline sequences and the CDR3 amino acid lengths of cloned antibody $\mathrm{H}$ and $\mathrm{L}$ gene sequences were analyzed for each subject. ( $F$ ) RBD (left) and spike (right) specific binding activities of five HuNAbs, including A6, B4, B7, B8 and C5, were measured by ELISA. (G) Neutralization activities of 5 HuNAbs against pseudotyped (left) and authentic (right) SARS-CoV-2 were determined in HEK 293T-ACE2 and Vero-E6 cells, respectively. HIV-1 specific HuNAb VRC01 served as a negative control. Each assay was performed in duplicates and the mean of replicates is shown with the standard error of mean (SEM). (H) The competition of four HuNAbs, including B4, B7, B8 and C5, with human soluble ACE2 for binding to SARS-CoV-2 RBD was measured by SPR. The curves show binding of ACE2 to SARS-CoV-2 RBD with (red) or without (black) pre-incubation with each HuNAb. 
Fig. 2
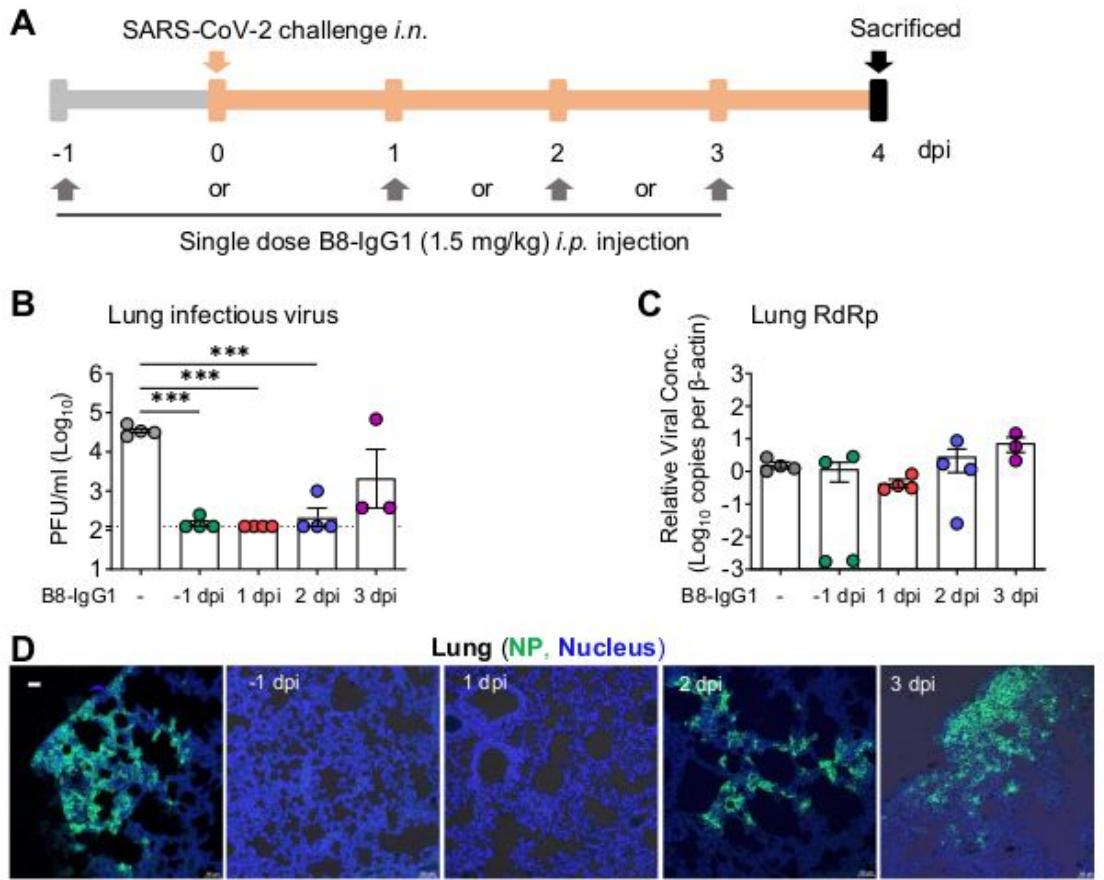

E

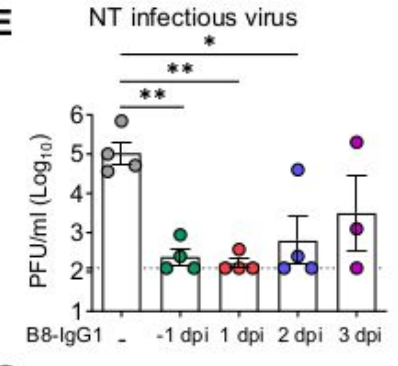

G

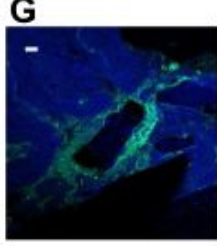

$\mathbf{F}$

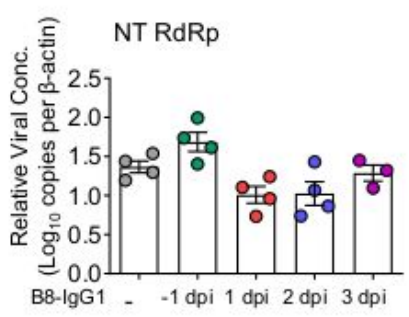

NT (NP, Nucleus)

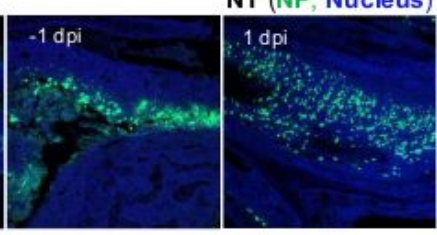

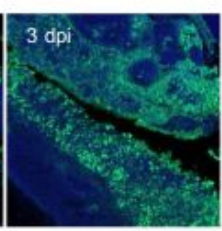

\section{Figure 2}

Pre- and post-exposure treatment of B8-IgG1 against SARS-CoV-2 in Syrian Hamster. (A) Experimental schedule. Four groups of hamsters (G1-G4) received intraperitoneally a single dose of $1.5 \mathrm{mg} / \mathrm{kg}$ of B8IgG1 at one day before infection (-1 dpi) for pre-exposure prophylaxis, and at day one (1 dpi), two (2 dpi) and three ( $3 \mathrm{dpi})$ post-infection for early treatment, respectively. Control hamsters $(\mathrm{G} 0, \mathrm{n}=4)$ received an isotypic control antibody at the same dose. On day 0 , each hamster was intranasally challenged with a 
dose of 105 PFU of SARS-CoV-2 (HKU-001a strain). All hamsters were sacrificed on 4 dpi for analysis. (B) Infectious virus (PFU) was measured in animal lungs by the viral plaque assay in Vero-E6 cells. The $\mathrm{PFU} / \mathrm{ml}$ concentration is shown in log-transformed units. (C) The relative viral RdRp RNA copies (normalized to $₫$-actin) were determined by RT-PCR in animal lungs. (D) Representative 100x images of infected lungs from each group, as determined by anti-NP immunofluorescence (IF) staining. The cell nuclei were counterstained with DAPI (blue). (E) Infectious virus (PFU) were measured in NT homogenates by the viral plaque assay as mentioned above. (F) Viral loads in NT homogenates of each group were determined by RT-PCR assay. The viral load data is shown in log-transformed units. (G) Representative 100x images of infected NT from each group as determined by anti-NP IF staining as mentioned above. Statistics were generated using one-way ANOVA tests. ${ }^{\star} p<0.05 ;{ }^{\star \star} p<0.01 ;{ }^{* \star \star} p<0.001$. 
Fig. $3 \mathrm{~A}$

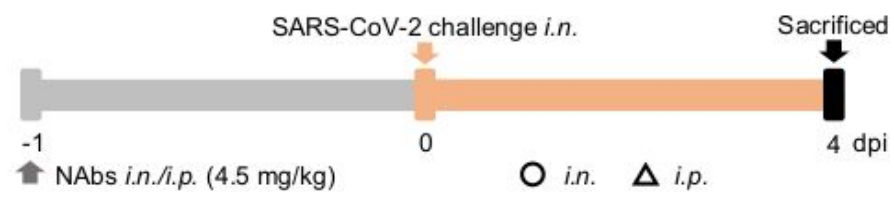

B

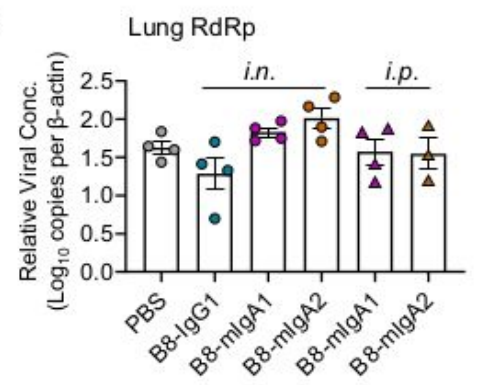

D

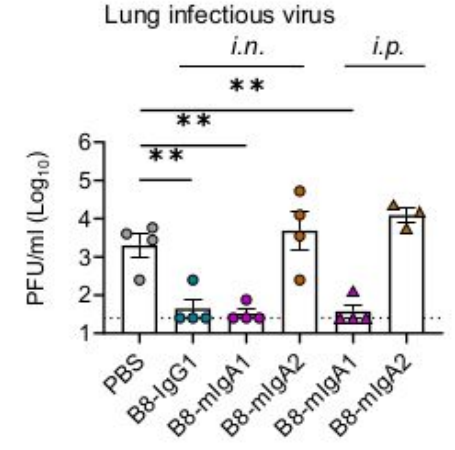

$\mathbf{F}$

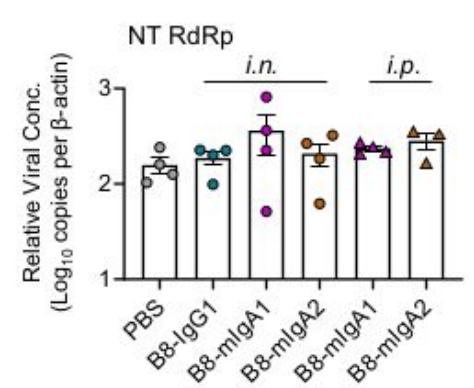

H

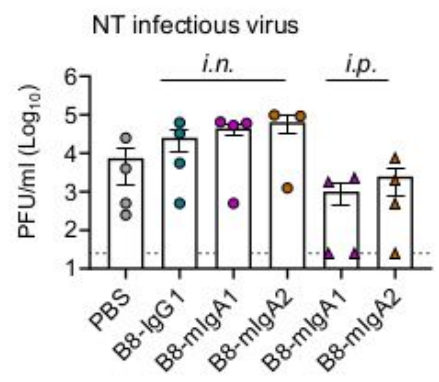

C

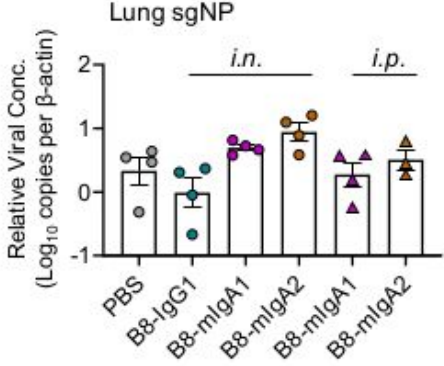

E

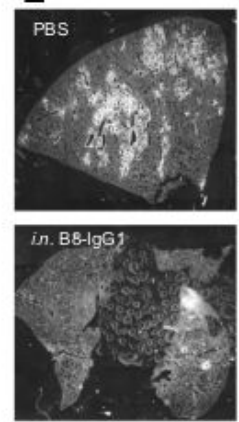

Lung (NP signal)
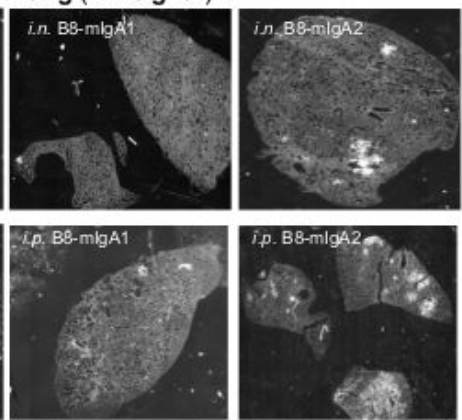

G
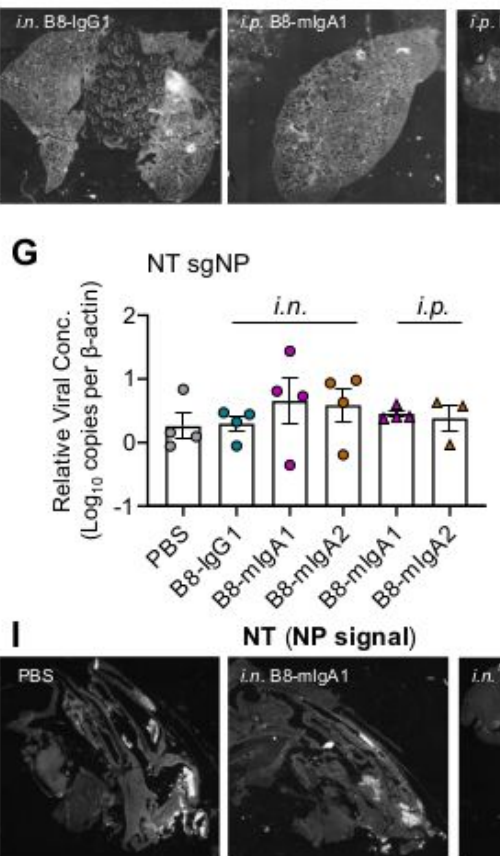

NT (NP signal)
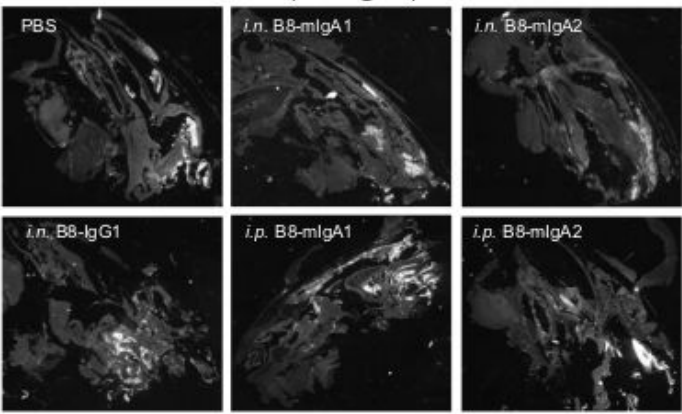

\section{Figure 3}

Pre-exposure treatment with monomeric B8-mlgA1 or B8-mlgA2 against SARS-CoV-2 infection in Syrian hamsters. (A) Experimental schedule. Five groups of hamsters ( $n=4$ per group) received a single dose of $4.5 \mathrm{mg} / \mathrm{kg}$ of B8-lgG1, B8-mlgA1 or B8-mlgA2 one day before viral challenge for pre-exposure prophylaxis by the intranasal route (circles) or the intraperitoneal route (triangles), respectively. Control hamsters $(n=4)$ received PBS. On day 0 , each hamster was intranasally challenged with a dose of 105 PFU of 
SARS-CoV-2, as mentioned in Fig. 2A. All hamsters were sacrificed on 4 dpi for analysis. (B) The viral RNA load, measured by relative RdRp RNA copy numbers (normalized to ß-actin) was determined by RT-PCR in animal lung homogenates. (C) The relative sub-genomic nucleocapsid (sgNP) RNA copy numbers (normalized to $ß$-actin) were determined by RT-PCR in animal lung homogenates. (D) Infectious virus (PFU) was measured in animal lung homogenates by the viral plaque assay in Vero-E6 cells. (E) Representative lung images of infected animals by scanning whole tissue section. The signal of SARSCoV-2 NP was shown in bright spots. (F) The relative viral RdRp RNA copy numbers (normalized to ßactin) were determined by RT-PCR in NT homogenates. (G) The relative sgNP RNA copy numbers (normalized to ß-actin) were determined by RT-PCR in NT homogenates. (H) Infectious virus (PFU) was measured in animal NT homogenates by the viral plaque assay in Vero-E6 cells. (I) Representative NT images of infected animals by scanning whole tissue section. The signal of SARS-CoV-2 NP was shown in bright spots. Log-transformed units are shown in (B) to $(H)$ except in (E). Statistics were generated using one-way ANOVA tests. ${ }^{*} p<0.05 ;{ }^{* *} \mathrm{p}<0.01$. 
Fig. 4 A

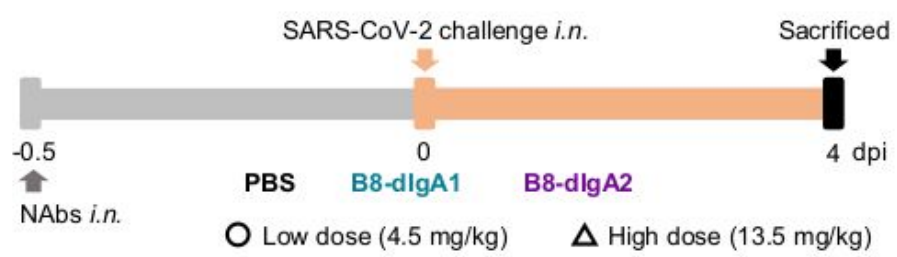

B

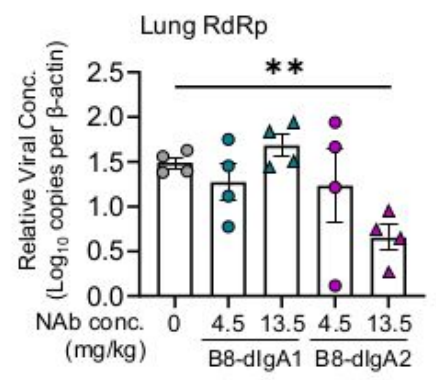

D

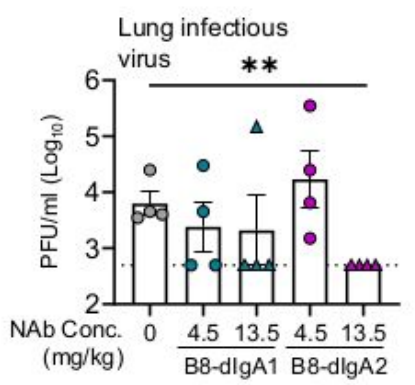

F

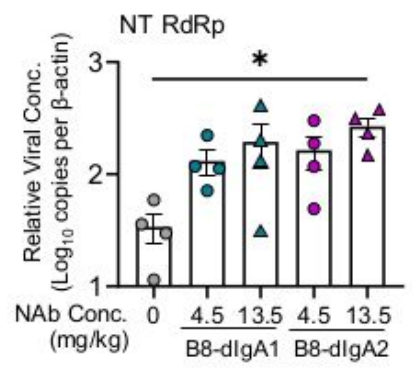

H

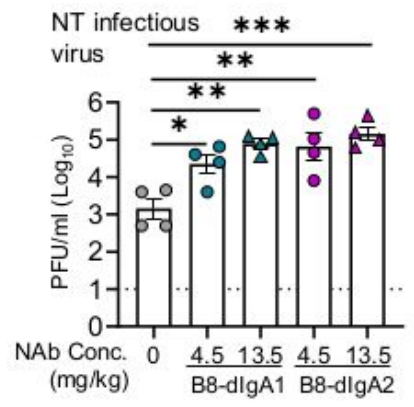

C

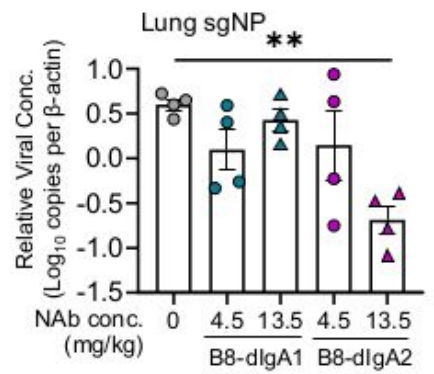

E
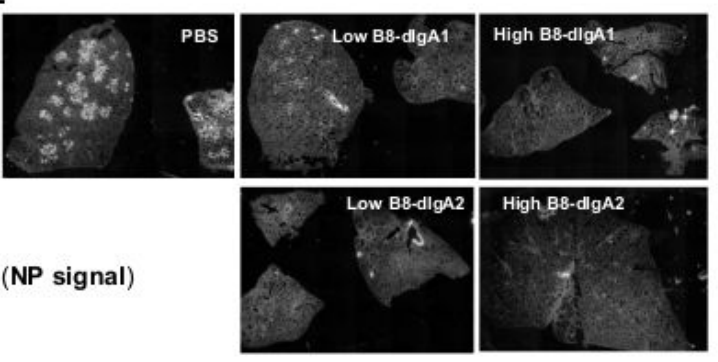

G

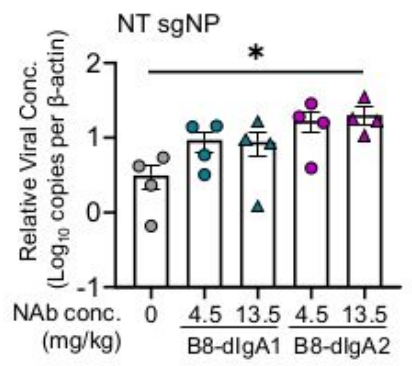

I
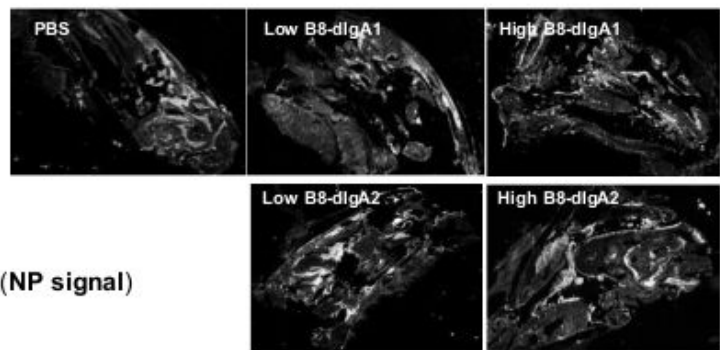

\section{Figure 4}

Pre-exposure treatment with dimeric B8-dlgA enhances SARS-CoV-2 infection in Syrian hamsters. (A) Experimental schedule. Four groups of hamsters ( $n=4$ per group) were inoculated intranasally with B8dlgA1 or B8-dlgA2, either at a low dose of $4.5 \mathrm{mg} / \mathrm{kg}$ or at a high dose of $13.5 \mathrm{mg} / \mathrm{kg}$, respectively, 12 hours before intranasal viral challenge. Another group of hamsters $(n=4)$ received PBS as control. On day 0, each hamster was intranasally challenged with a dose of 105 PFU of SARS-CoV-2 as described in Fig. 
2A. All hamsters were sacrificed on $4 \mathrm{dpi}$ for analysis. Data represent a presentative experiment from three independent experiments. (B) The relative viral RdRp RNA copy numbers (normalized to $B$-actin) were determined by RT-PCR in animal lung homogenates. (C) The relative viral sgNP RNA copy numbers (normalized to B-actin) were determined by RT-PCR in animal lung homogenates. (D) Infectious virus (PFU) was measured in animal lung homogenates by the viral plaque assay in Vero-E6 cells. (E) Representative lung images of infected animals by scanning whole tissue section. The signal of SARSCoV-2 NP was shown in bright spots. (F) The relative viral RdRp RNA copy numbers (normalized to ßactin) were determined by RT-PCR in NT homogenates. (G) The relative viral sgNP RNA copies (normalized to ß-actin) were determined by RT-PCR in NT homogenates. $(H)$ Infectious virus (PFU) was measured in NT homogenates by the viral plaque assay in Vero-E6 cells. (I) Representative NT images of infected animals by scanning whole tissue section. The signal of SARS-CoV-2 NP was shown in bright spots. Log-transformed units are shown in $(B)$ to $(H)$ except in $(E)$. Statistics were generated using oneway ANOVA tests. ${ }^{*} \mathrm{p}<0.05 ;{ }^{* *} \mathrm{p}<0.01$. 
Fig. 5

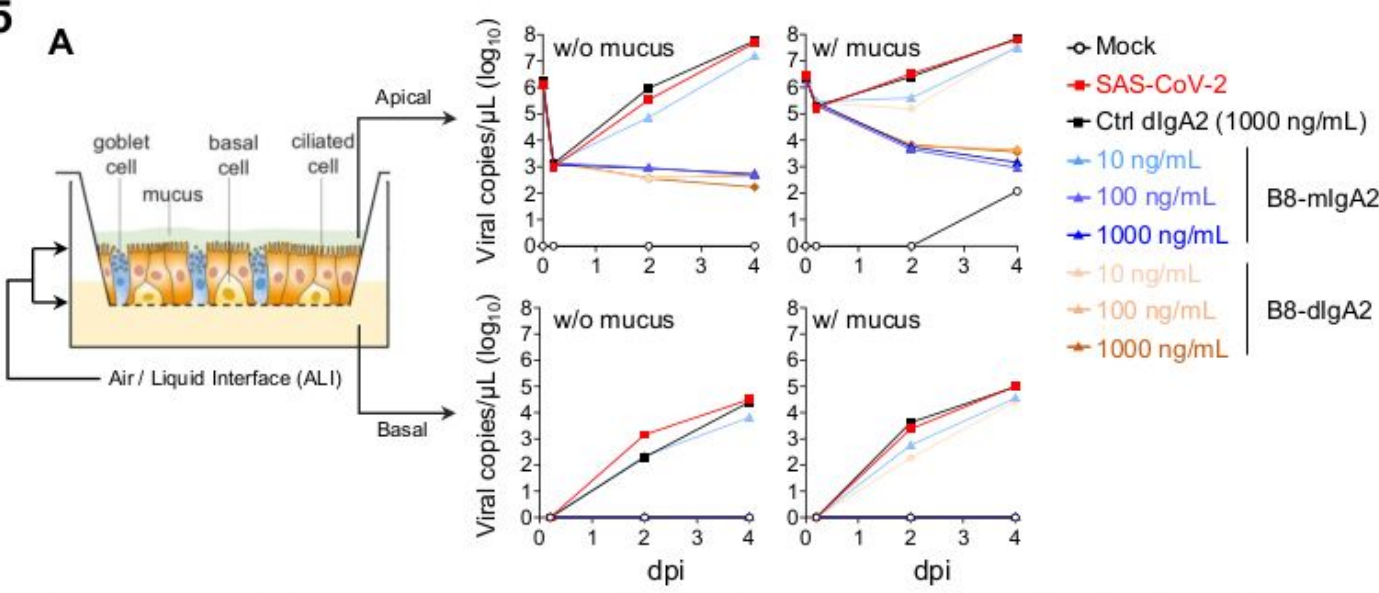

B

B8-dlgA2

SARS-CoV-2

B8-dlgA2 + SARS-CoV-2
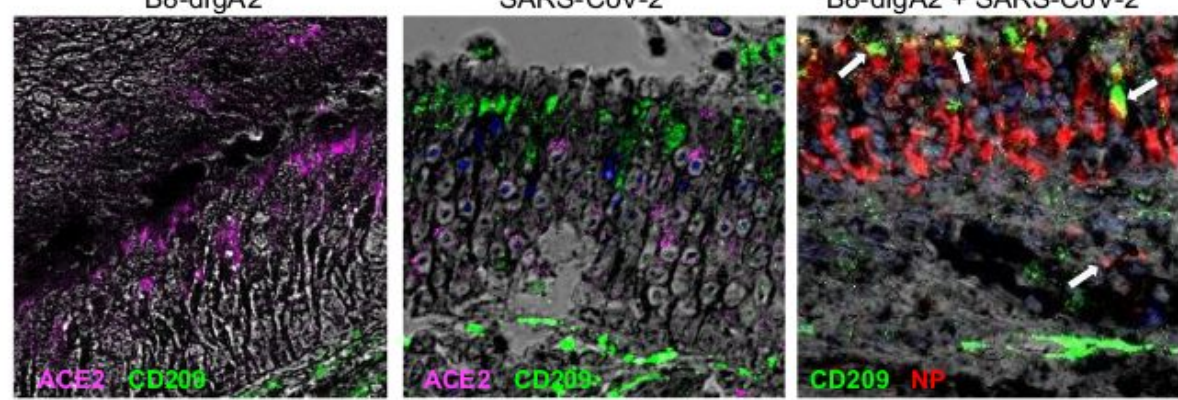

C
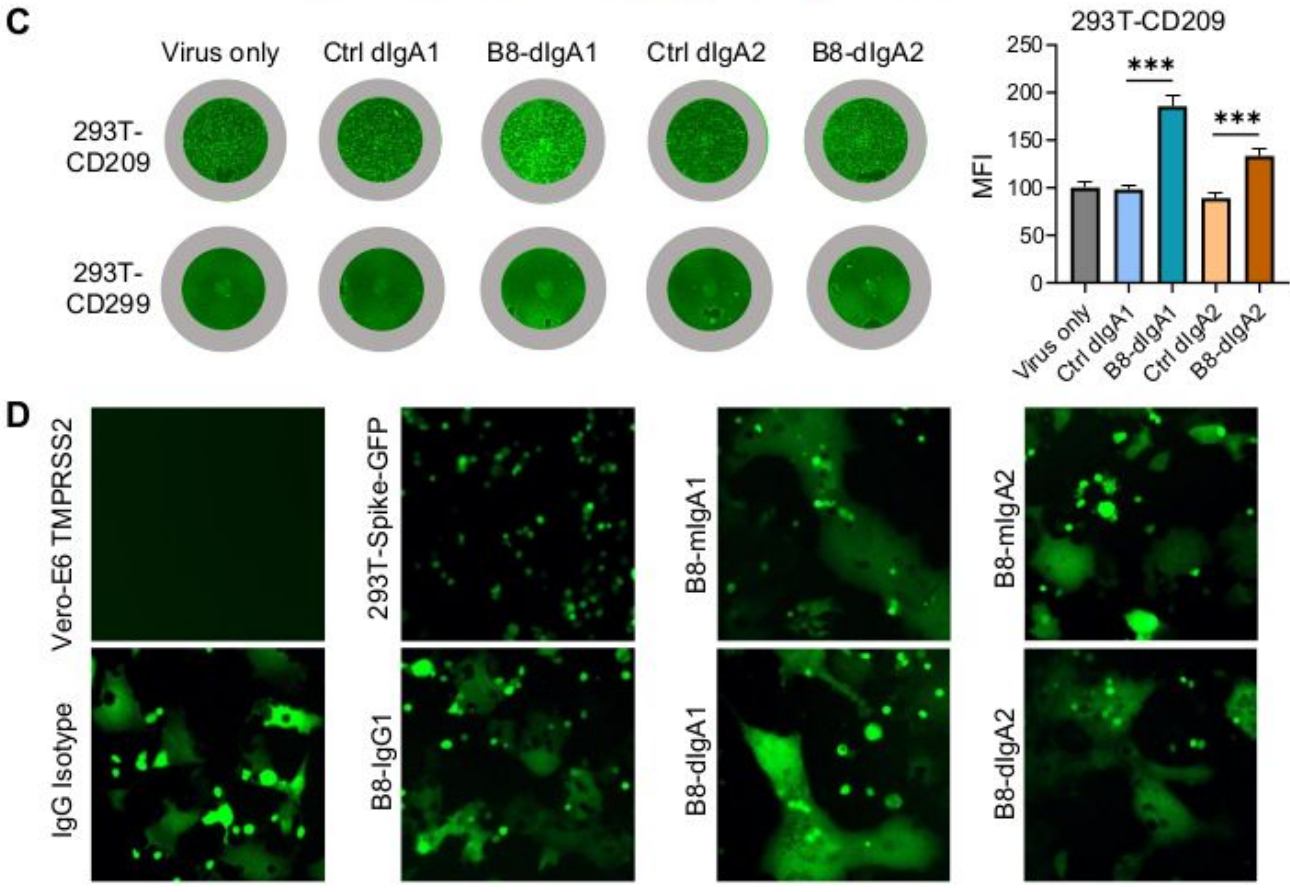

\section{Figure 5}

B8-dlgA1 and B8-dlgA2 enhance SARS-CoV-2 infection via CD209. (A) The effects of B8-dlgA2 on SARSCoV-2 infection in the MucilAirTM model, consisting of primary human nasal epithelial cells but no DCs. B8-mlgA2 or B8-dlgA2 were pre-incubated at doses of 10,100 , and $1000 \mathrm{ng} / \mathrm{ml}$, respectively, in the apical compartment with or without mucus for 1 hour, before adding 104 PFU of SARS-CoV-2 (BetaCoV/France/IDF00372/2020) for 4 hours. The viral RNA loads were measured by RT-PCR in both the 
apical and basal compartments and are shown in log-transformed units. (B) Representative confocal images (400x) of olfactory epithelium in NT showed the expression of CD209 (DC-SIGN) in green and ACE2 in magenta by immunohistochemical staining of experimental hamsters treated with B8-dlgA2 without (left) or with (middle and right) SARS-CoV-2 infection. Color-coding indicates specific antibodies used for double staining. Infected CD209+ cells are visualized in yellow as indicated by arrows (right). (C) The CD209 or CD299 overexpressed-HEK 293T cells were pre-treated for 6 hours with $10 \mathrm{ng} / \mathrm{ml}$ of B8dlgA1 or B8-dlgA2 or control dlgA1 or control dlgA2 or PBS, respectively, prior to SARS-CoV-2 infection (MOI: 0.05). Two days after infection, SARS-CoV-2 NP expression (green) was quantified by the mean fluorescence intensity (MFI) after anti-NP IF staining. Statistics were generated using student-t tests. ${ }^{*} p<0.05 ;{ }^{* *} p<0.01 ;{ }^{* *} p<0.001$. (D) The effects of B8 antibodies on cell-cell fusion. 293T cells cotransfected with SARS-CoV-2 spike and GFP were pre-treated with 100x the IC90 dose of B8-IgG1, B8mlgA1, B8-mlgA2, B8-dlgA1, B8-dlgA2 or and IgG isotypic control for 1 hour, respectively. Vero-E6 cells transfected with TMPRSS2 were then added to the treated 293T-spike-GFP cells and co-cultured for 48 hours. Cell-cell fusion was imaged under a fluorescence confocal microscope at the 50x magnification. 
Fig. 6

A

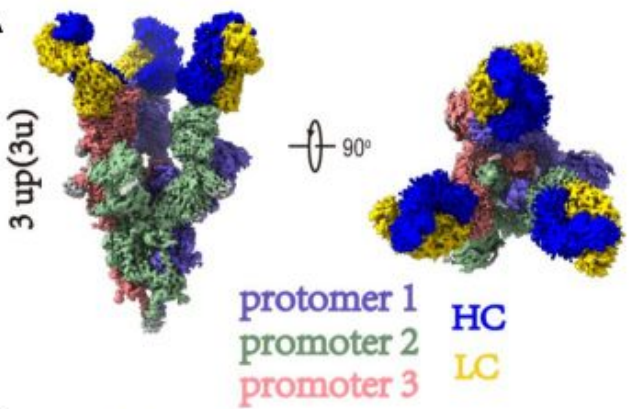

C

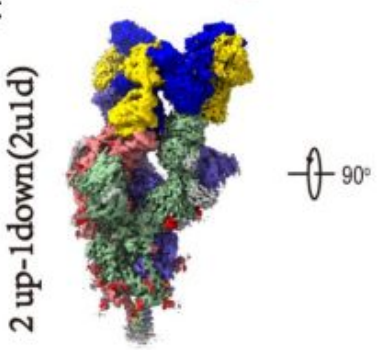

E
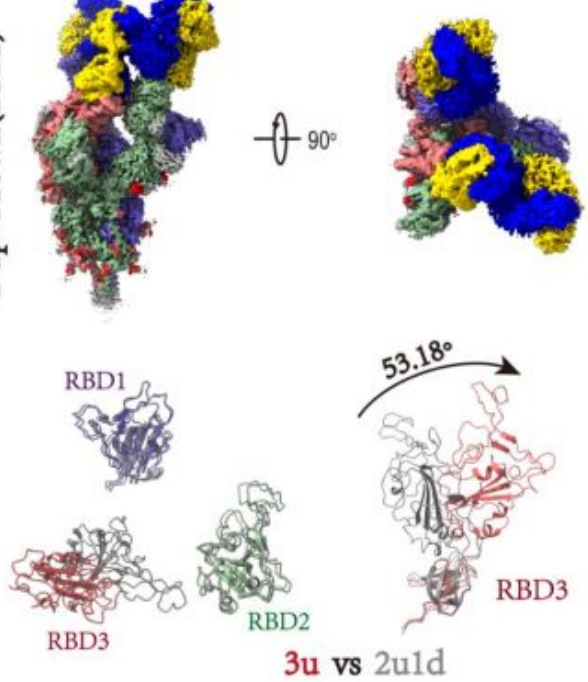

B

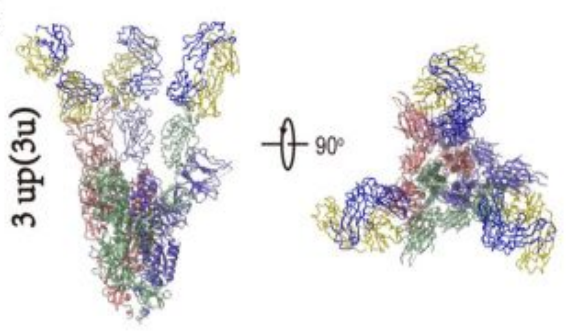

D

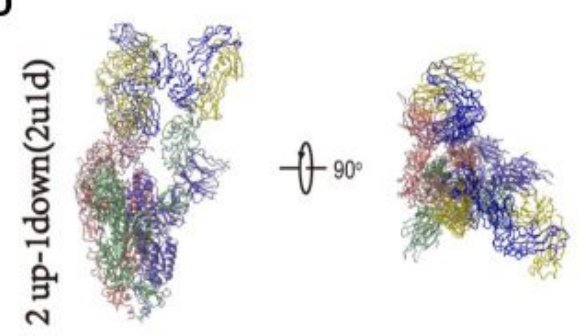

G
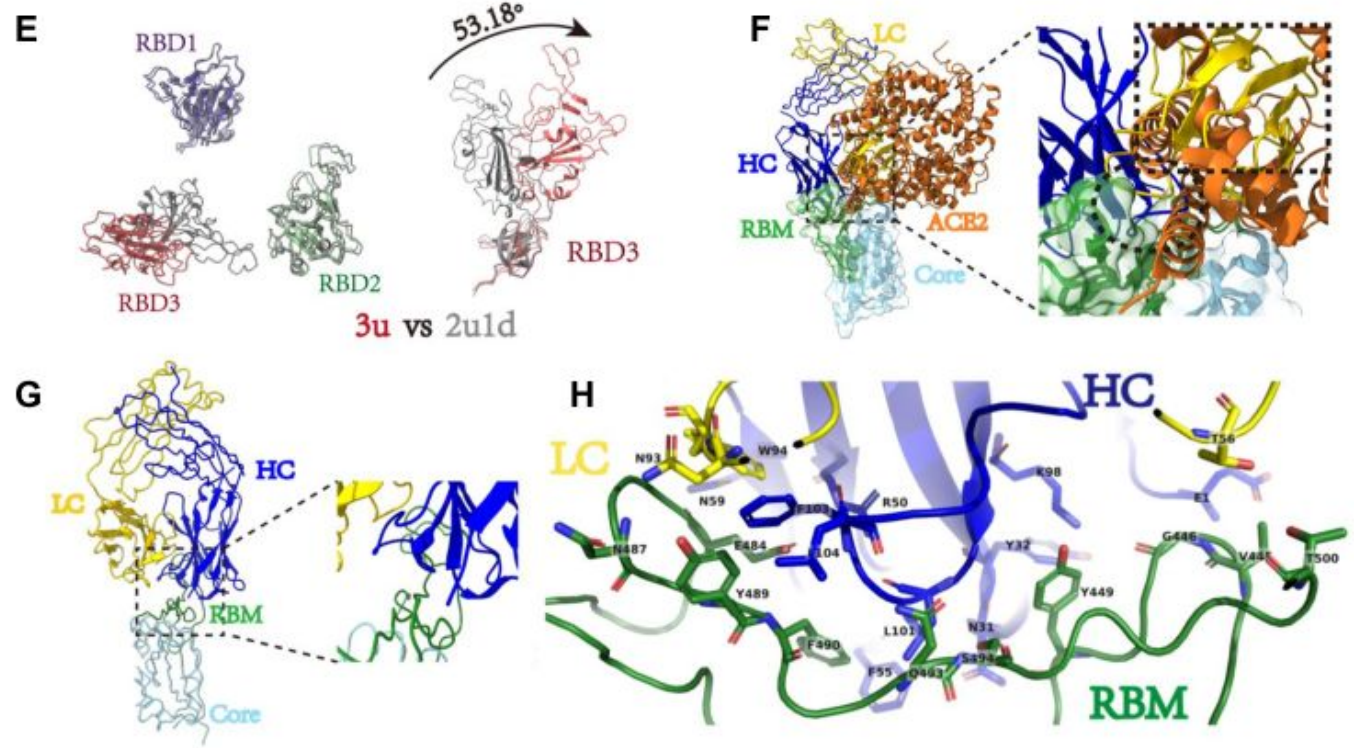

\section{Figure 6}

Cryo-EM structures of the SARS-CoV-2 S trimer in complex with B8-Fabs. (A) Side and top views of the Spike-B8 3u cryo-EM map showing 3 up RBDs each bound with a B8 Fab. Protomer 1, 2, and 3 are shown in slate blue, dark sea green, and India red, respectively. Heavy chain and light chain of the B8-Fab are in blue and gold, respectively. This color scheme was used throughout panels (A)-(E). (B) Side and top views of the Spike-B8 3u cryo atomic model. (C) Side and top views of the Spike-B8 2u1d cryo-EM map showing 
two up RBDs up (RBD-1 and RBD-2) and one RBD down (RBD-3), each bound to a B8-Fab. (D) Side and top views of the Spike-B8 2u1d cryo atomic model. (E) Structural comparation of RBDs between Spike-B8 $3 u$ (different colors) and Spike-B8 2u1d (gray). (F) ACE2 (chocolate color, PDB: 6M0J) may clash with the heavy chain (blue) and light chain (gold) of the B8-Fab. ACE2 and the Fab share overlapping epitopes on the RBM (dotted black circle), and the framework of the B8-VL appears to clash with ACE2 (dotted black frame). The RBD core and RBM are shown in light sky blue and green, respectively. (G) Atomic model of an RBD-B8 complex portion in cartoon mode, shown with the same color scheme as in $(F)$. (H) The residues involved in interactions between B8 and the RBM. The heavy and light chain of the B8-Fab are in blue and gold, respectively. The RBM is shown in green.

\section{Supplementary Files}

This is a list of supplementary files associated with this preprint. Click to download.

- Supplementaryfiguresandtables20210921NatureMicro.pdf 